\title{
Field validation and benchmarking of a cloud shadow speed sensor
}

\author{
P. Kuhn ${ }^{1, *}$ \\ Paseo de Almería 73, 04001 Almería, Spain
}

M. Wirtz ${ }^{1}$, S. Wilbert ${ }^{1}$, J. L. Bosch ${ }^{2}$, G. Wang ${ }^{3}$, L. Ramirez ${ }^{4}$, D. Heinemann ${ }^{5}$, R. Pitz-Paal ${ }^{6}$

\begin{abstract}
With ramp rate regulations for photovoltaic plants being discussed in many countries, the speed of clouds has gained significant importance lately. Besides, measuring cloud velocities and directions is of interest for validations of numerical weather predictions and solar nowcasting systems. Recently, the Cloud Shadow Speed Sensor (CSS) was developed and validated in San Diego for low cumulus clouds. In this publication, the CSS is studied under different weather and cloud conditions in the desert of Tabernas in southern Spain. Furthermore, a novel shadow camera based low-cost, low-maintenance approach to determine cloud shadow motion vectors is presented and used as a reference to benchmark the CSS. In comparison, the absolute velocities derived from the CSS and the shadow camera on 59 days for \pm 5 min temporal medians show deviations of

\footnotetext{
* Corresponding author

Email address: Pascal.Kuhn@dlr.de (P. Kuhn)

${ }^{1}$ German Aerospace Center (DLR), Institute of Solar Research, Paseo de Almería 73, 04001 Almería, Spain.

${ }^{2}$ Departamento de Ingeniería Eléctrica y Térmica, Universidad de Huelva, Campus de La Rábida, Carretera de Palos de la Frontera S/N 21071 La Rábida, Palos de la Frontera (Huelva)

${ }^{3}$ Dept of Mechanical and Aerospace Engineering, UCSD Center for Energy Research, University of California, 92093-0411 La Jolla, USA.

${ }^{4}$ CIEMAT, Energy Department - Renewable Energy Division. Av. Complutense, 40, 28040 Madrid, Spain.

${ }^{5}$ Energy Meteorology Unit, Energy and Semiconductor Research Laboratory, Institute of Physics - Oldenburg University, 26111 Oldenburg, Germany.

${ }^{6}$ German Aerospace Center (DLR), Institute of Solar Research, Linder Höhe, 51147 Cologne, Germany.
} 
RMSD $2.1 \mathrm{~m} / \mathrm{s}(28.0 \%)$, MAD $1.2 \mathrm{~m} / \mathrm{s}(15.7 \%)$ and a bias of $-0.2 \mathrm{~m} / \mathrm{s}(2.8 \%)$. Deviations of the cloud shadow direction are RMSD $47.9^{\circ}$ (26.6\%), MAD 25.3 $(14.0 \%)$ and bias $3.7^{\circ}(2.0 \%)$. An adaption of the CSS software yields $91 \%$ more measurements on 59 days in comparison to the previously used algorithms at the expense of reduced accuracies, both for the measured velocities and for the measured directions.

The CSS and the novel shadow camera based reference system enable longtime, low-maintenance ground measurements of cloud shadow speeds, which were previously not available. The distinct advantages and limitations of the two systems are discussed. In addition to the comparisons between the shadow camera system and the CSS on 59 days, the detection rates of the CSS are classified and measured on 223 days by analyzing CSS radiometer signals. Depending on the shading strength and shading durations, detection rates vary between $3.7 \%$ and $21.6 \%$. Furthermore, the basic assumption as well as possible correction approaches of the linear cloud edge - curve fitting method are studied.

The CSS was found to be a robust tool with great potential. However, optically thin clouds with diffuse edges pose a challenge and the detection rate leaves room for improvements. The newly developed shadow camera system provides more measurements which scatter less but needs certain geographical requirements. The shadow camera is found to be a feasible validation tool for cloud (shadow) motion vectors.

Keywords: Cloud shadow speed sensor, cloud speed, shadow camera system

\section{1. Introduction}

$2 \quad$ Obtaining reference motion vectors of clouds is relevant for the optimization

3 and validation of all-sky imager based nowcasting systems (Kuhn et al. 2017a)

4 as well as numerical weather predictions (NWP) and satellite-based weather

5 forecasts (Molteni et al. (1996), Klein and Jakob (1999), Tomassini et al. (1999)).

- In addition to that, the rapid growth of solar power generation with its inherent 
7 variability calls for solar forecasting tools, which can predict shading events.

8 Recently, ramp rate regulations (Lave et al. (2013), Marcos et al. (2014), Chen

9 et al. (2017)) in several countries with high solar grid penetrations have further

10 stressed the need of cloud speed measurements. The Cloud Shadow Speed

11 Sensor (CSS) can be used to derive such cloud motion vectors and can be a part

12 of a camera-based solar nowcasting system (Wang et al. 2016). A singular all-

13 sky imager can measure angular speeds of clouds, but cannot provide absolute

14 speeds in $[\mathrm{m} / \mathrm{s}]$.

15 The CSS, pictured in Fig. 1, was developed and presented in Fung et al.

16 (2013). Previous validations, both under laboratory conditions and in-field,

17 have been conducted (Fung et al. 2013). However, the variability of clouds

18 and the complexity of the weather vary for different locations. For instance, in

19 San Diego (USA), where the CSS was previously validated, cloud heights rarely

20 exceed $1000 \mathrm{~m}$ (Wang et al., 2016).

21 In this publication, the CSS is compared to a novel shadow camera reference 22 system on 59 days at the Plataforma Solar de Almería (PSA) in southern Spain.

${ }_{23}$ In southern Spain, a wide range of cloud speeds, heights and clouds of various

24 classes is observed (Killius et al. (2015), Kuhn et al. (2017a)). Investigating

25 and benchmarking the performance of the CSS in this complex meteorological

26 environment gives insights into its general applicability. In addition to the

${ }_{27}$ comparison against a shadow camera on 59 days, the detection rate of the CSS

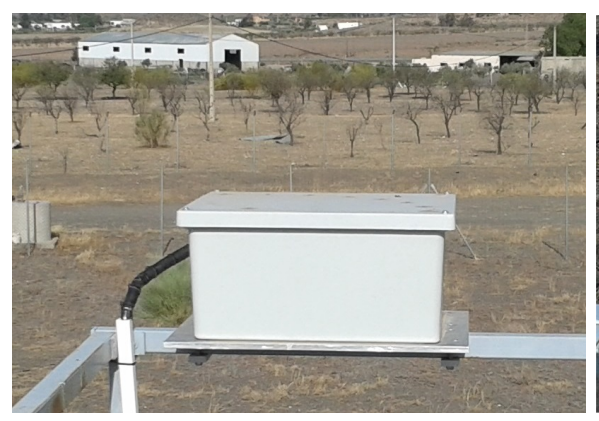

(a)

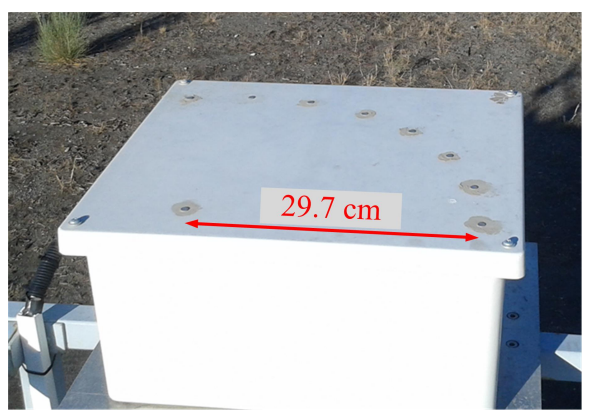

(b)

Figure 1: The Cloud Shadow Speed Sensor (CSS) at PSA, Spain. 
43 method are studied.

\section{2. The Cloud Shadow Speed Sensor}

\section{2.1. Working principle}

46 The working principle of the CSS, developed by Fung et al. (2013), is based

47 on methods for determining cloud motion vectors with an array of irradiance 48 sensors (Bosch and Kleissl (2013), Bosch et al. (2013), Schenk et al. (2015)). It

49 consists of nine uncalibrated photodiode pyranometers, which are sampled at

so a frequency of $667 \mathrm{~s}^{-1}$. Eight of these sensors are placed in a circular arc of $51105^{\circ}$ with a radius of $29.7 \mathrm{~cm}$ around the ninth sensor (see Fig. 1). In order to 52 measure the speed and direction of a cloud shadow, the CSS must be directly 53 shaded. If the shadow of a cloud passes the CSS, the sensors detect ramps at 54 slightly different times. This way, both the speed and the direction of the clouds

55 is determined. Due to the high frequency, the distances of the sensors can be 
67 2.2. Software adaptions of the CSS CSS with and without these adaptions.

\section{Increasing the detection rate}

small, which enabled a very compact design. Overall material costs are specified to be approximately 400 US- $\$$ (Wang et al. 2016).

The CSS does not need regular cleaning as the working principle is based on relative deviations, not absolute irradiance measurements. As experienced over more than two years of active service, this user-friendly maintenance routine was found to hold even in the harsh conditions of the desert of Tabernas (Almería, Spain). Although not cleaned, the CSS data are checked daily, e.g. to detect constantly shaded sensors due to bird excrements. Luckily, such an event did not occur yet. Based on this differential approach, the CSS is able to determine the motion vectors of cloud shadows, not directly the motion vectors of the clouds. However, these vectors deviate only insignificantly (Fung et al., 2013).

During this comparison campaign, no hardware adjustments were conducted on the CSS. Suggestions for hardware improvements are mentioned in the conclusion. However, the evaluation method of the CSS is scrutinized and adapted. All comparisons to the shadow camera measurements will be conducted on the

In the first step of the evaluation algorithm, the CSS filters its data and it does not provide cloud speed measurements if certain criteria are not met. In any case, however, the raw data is stored. The filtering as implemented in Fung et al. (2013) and Wang et al. (2016) is based on a second order error metric (presented in the following), which results in a low number of calculated cloud motion vectors in relation to the total number of shading events.

The algorithm used for the cloud motion measurements itselves and described in Wang et al. (2016) is the LCE - curve fitting algorithm, which determines the maximum cross-correlation coefficient $R_{i j}$ of each pair of signals and records the associated time shift $\Delta t_{i, j}$ for the sensor pair consisting of sensor $i$ and $j$ corresponding to this maximum cross-correlation. Due to the setup of 


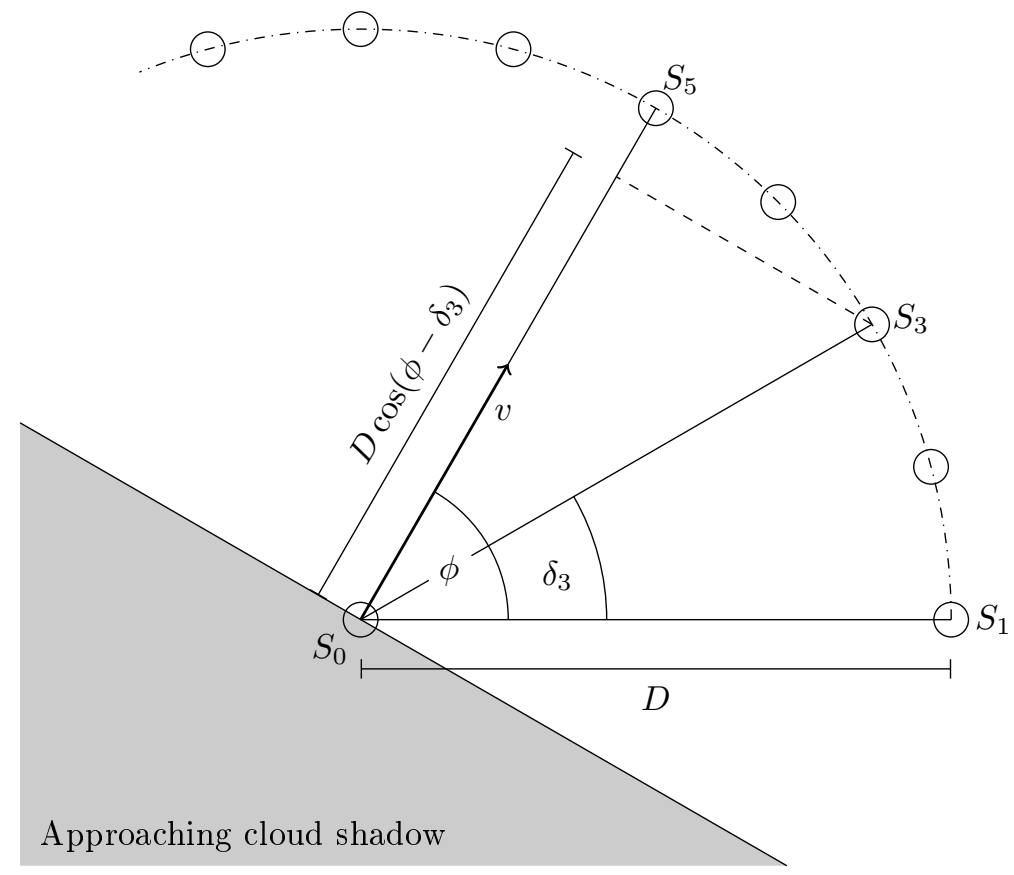

Figure 2: Depicted in the bottom-left corner is a shadow approaching the CSS with a speed $v$ and a direction $\phi$. Sensor $S_{0}$ is shaded first, sensor $S_{1}$ is shaded $\frac{D}{v} \cos (\phi)$ after $S_{0}$. Then sensor $S_{3}$ is shaded $\frac{D}{v} \cos \left(\phi-\delta_{3}\right)$ and $S_{5} \frac{D}{v}$ after $S_{0}$. Based on these time differences, the motion vector of the shadow can be calculated.

${ }_{85}$ the CSS, there are $\#(i \circ j)=\# \alpha=12$ sensor pairs. Based on the time shifts of

s6 these sensor pairs, the speed is calculated. The method will be briefly described

${ }_{87}$ here and is explained in detail in Wang et al. (2016).

s8 In Fig. 2, an example situation is shown. Coming from the bottom-left, a 89 shadow is sequentially shading the sensors. The trigonometric relation visualized 9o in Fig. 2 holds for all cloud edge directions as the cloud speed is assumed to be 91 perpendicular to the cloud edge. Deviations caused by this this assumption are 92 studied in section $\mathrm{A}$

93 The residuum of the cosine fit $\Gamma$ acts as a filter (equ. 1).

${ }_{94} \quad \Gamma=1-\frac{\sum_{\alpha=1}^{12}\left(t_{\alpha, F i t}(\phi, v)-t_{\alpha}\right)^{2}}{t_{R M S}}$ 
It is calculated with $t_{\alpha, F i t}(\phi, v)$ being the time shift according to the calculated cosine fit, $t_{\alpha}$ being the measured time shift and $t_{R M S}$ being the quadratic scatter of the time shifts according to equ. 2 .

$$
t_{R M S}=\sum_{\alpha=1}^{12}\left(t_{\alpha}-\frac{1}{12} \sum_{\alpha=1}^{12} t_{\alpha}\right)^{2}
$$

If the average of the maximum cross-correlation coefficients $R_{i j}$ is less than 0.9 or the residuum $\Gamma$ of the cosine curve fit is less than 0.9 , the cloud motion vector will not be computed. A small $R_{i j}$ is likely a result of an erroneous measurement or dynamically changing clouds. Similar, a small $\Gamma$ indicates poor curve fitting and therefore an unreliable result. Based on these two criteria, measurements are rejected. The calculation of the cosine fit is based on a least square approach (LSQ). This approach, presented in Wang et al. (2016), is highly sensitive towards outliers and thus rejects many measurements.

In order to reduce the influence of outliers towards the cosine fit, several regression models such as the least square method (LSQ, Wang et al. (2016)), the least absolute deviation method (LAD, Bloomfield and Steiger (2012)), the least trimmed squares method (LTS, Giloni and Padberg (2002), Mount et al. (2014)) and the least median of squares method (LMS, Rousseeuw (1984)) were studied. All methods are discussed in detail in the literature (Rousseeuw and Croux (1993), Huber (2009) ) and will not be introduced here. Considering 347023 measuring intervals on 223 days, the LSQ method obtains 5830 cloud motion vectors (speed and direction). The LAD method obtains 8034, the LTS method 17334 and the LMS method 21535 motion vectors. The LTS method is found to have the least deviations in comparison to the LSQ method and yields $197 \%$ more measurements on 223 days (91\% more measurements on the 59 days which could be temporally matched to shadow camera measurements as considered in section 4.2 and section 4.3. The CSS measurements derived from both the LSQ and the LTS method will be compared to shadow camera measurements. In section 4.4 the determination of the detection rate is presented.

Lowering the thresholds of the LSQ method can also be used to obtain more measurements. However, these additional measurements are far less accurate if 


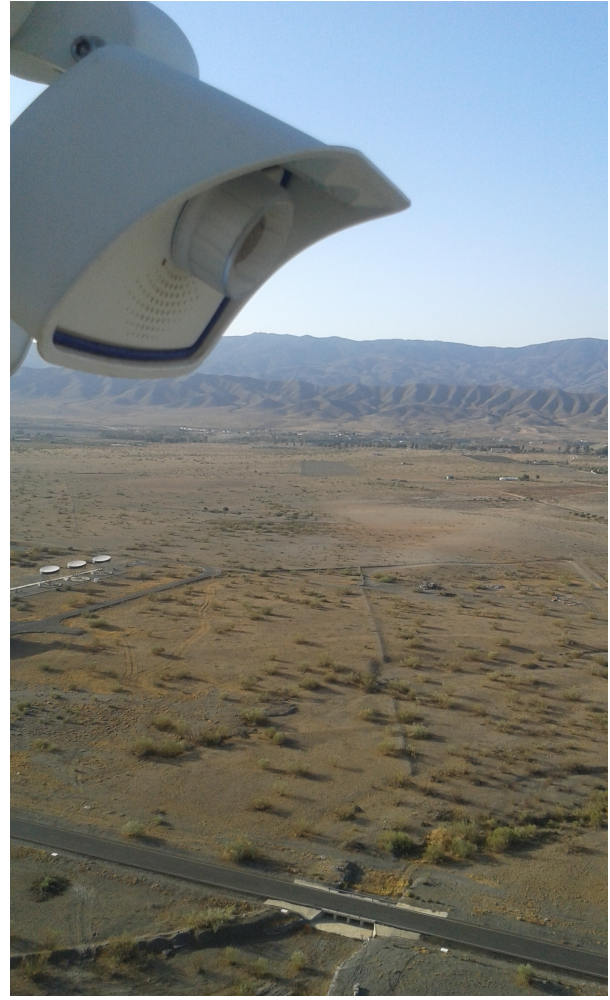

Figure 3: One of the six shadow cameras overlooking the PSA from top of a tower (CIEMAT CESA-I), $87 \mathrm{~m}$ above the ground.

133 (2006)) and external (via GPS reference points) orientation, an orthoimage is

${ }_{134}$ calculated (Fig. 4 p). In this orthoimage, the dimensions of all pixels are known 


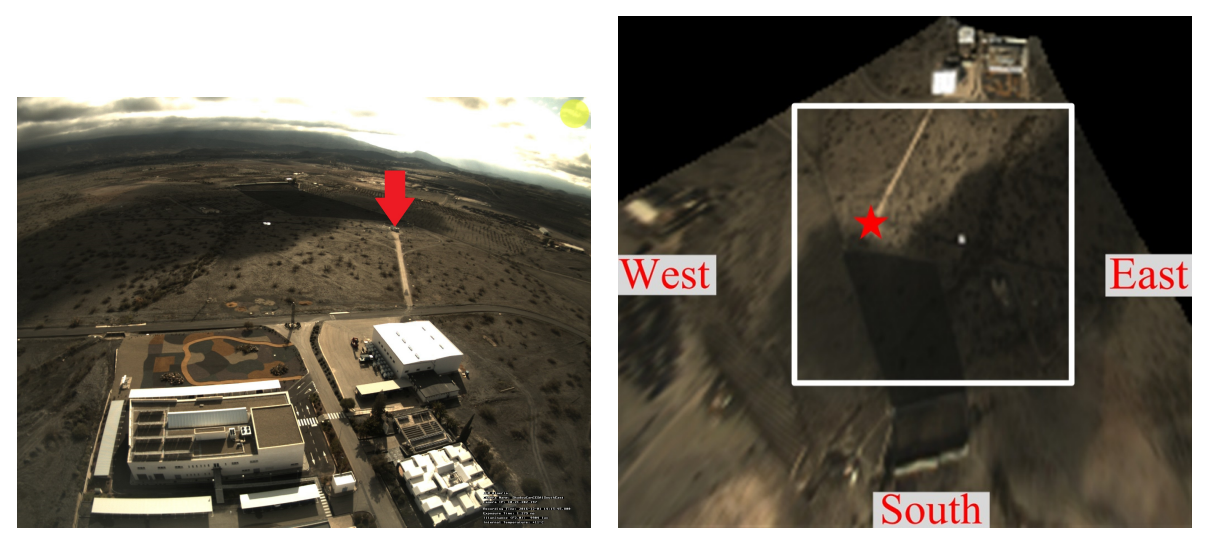

Figure 4: Left: raw image of the used shadow camera. The arrow marks the position of the CSS. Right: undistorted raw image as projected on a ground model. The star marks the position of the CSS. The white frame depicts the $525 \mathrm{~m} \times 525 \mathrm{~m}$ large area in which cloud shadow speeds are determined.

150 151

in $[\mathrm{m}]$. From three concurrent orthoimages and a novel differential approach, cloud speeds and cloud directions are resolved. Due to the viewing geometry, pixels imaging areas far away from the camera's position are distorted (see e.g. bottom-left in Fig. $4 \mathrm{~b}$ ). In order to derive robust cloud motion vectors, only a quadratic area of $105 \times 105$ pixels $(525 \mathrm{~m} \times 525 \mathrm{~m})$ within the orthoimage is considered.

The approach to derive cloud (shadow) motion vectors is visualized in Fig. 5 Three subsequent cropped orthoimages corresponding to the timestamps t, t- $\Delta t$ and t-2 $2 t$ are converted to grayscale and two difference images $d_{i}$ are derived. The first difference image $d_{1}$ is the absolute of the subtraction of the image $t$ and image $\mathrm{t}-\Delta t$. The second difference image $d_{2}$ is the absolute of the subtraction of the images $\mathrm{t}-\Delta t$ and $\mathrm{t}-2 \Delta t$. The approach is given in equ. 3 and equ. 4 with $\Delta t$ being $15 \mathrm{~s} . x$ and $y$ are the pixel coordinates in the cropped grayscale orthoimages $i_{\text {ortho }}$.

$$
d_{2}(x, y)=i m_{\text {ortho }}(x, y, t-\Delta t)-i m_{\text {ortho }}(x, y, t-2 \Delta t)
$$




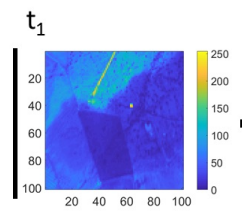

$-\Delta \mathrm{t}$

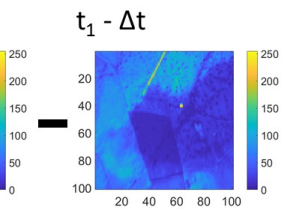

$d_{1}(x, y)$

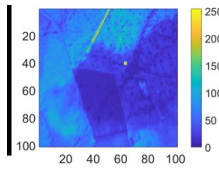

$t_{1}-2 \Delta t$
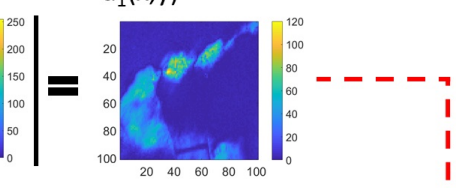

$d_{2}(x, y)$
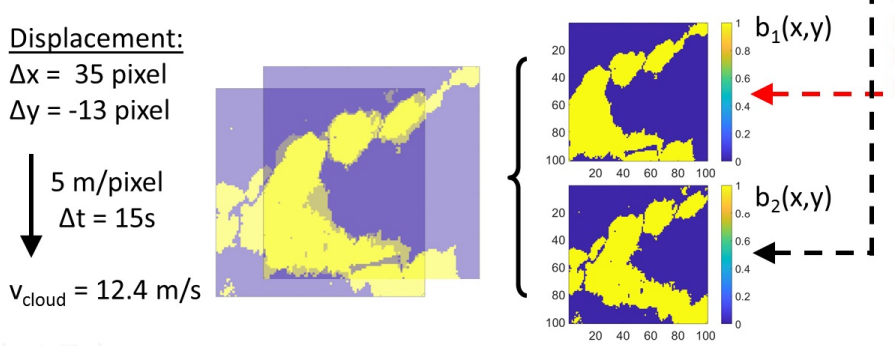

Figure 5: Shadow camera deriving cloud motion vectors: from three subsequent cropped and grayscale-converted orthoimages, difference images $d_{i}$ are calculated. Via an empirically found threshold, binary difference images $b_{i}$ are derived. These two difference images are then matched using cross-correlation. For the example situation depicted here (2016-12-01, 14:15:15 h - 14:15:45 h, UTC +1 ), a displacement of $\Delta x=35$ pixel and $\Delta y=-13$ pixel is calculated. This corresponds to a shadow velocity of $12.4 \mathrm{~m} / \mathrm{s}$.

The difference images are converted into binary images $b_{i}$ by an empirically found threshold (dashed arrows in Fig. 5). The pixel displacements $\Delta x$ and $\Delta y$ between the two binary difference images $b_{i}$ is obtained by the normalized 2-D cross-correlation approach presented in Huang et al. (2012) (see Fig. 5 bottom row). From the displacement vector, the cloud shadow speed can be derived using equ. 5 .

$$
v=\frac{\sqrt{(\Delta x)^{2}+(\Delta y)^{2}}}{\Delta t} \times k_{S C}
$$

Caused by technical limitations, the shadow camera can reliably resolve cloud motion vectors up to $17.5 \mathrm{~m} / \mathrm{s}$. The limiting factor is a result of the temporal resolution of $\Delta t=15 \mathrm{~s}$. This image acquisition rate is chosen to limit the amount of produced data. The camera itself can take up to 25 images per 


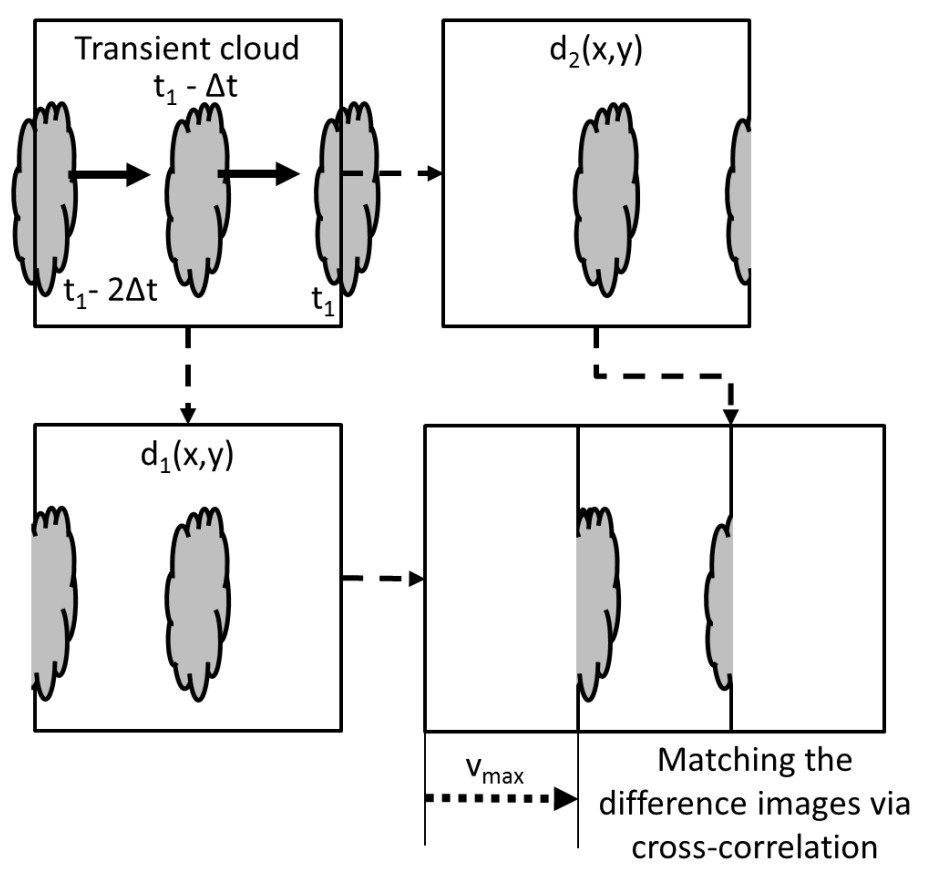

Figure 6: Visualization of the maximum resolvable velocity $v_{\max }$ : due to storage limitations, imposing a low image acquisition rate, the used shadow camera can reliably resolve cloud motion vectors up to $17.5 \mathrm{~m} / \mathrm{s}$.

second. The maximum velocity is calculated with equ. 6 and visualized in Fig. 6

$$
v_{\max }=\frac{N k_{S C}}{2 \Delta t}=17.5 \mathrm{~m} / \mathrm{s}
$$

Equation 6 is derived by looking at a cloud crossing the area under consideration in parallel to its borders (see Fig. 6). The quadratic imaged area has edge lengths of $N k_{S C}=105$ pixel $\cdot 5 \mathrm{~m} /$ pixel $=525 \mathrm{~m}$. A cloud entering the imaged area at time $t-2 \Delta t$ and leaving it at time $t$ results in a first (absolute) difference image $d_{1}$ with detected movements at a border and in the center. Similarly, the second difference image $d_{2}$ detects movements in the center and at the adjacent border. The matching via cross-correlation effectively divides the area by two, which this way defines the maximum resolvable velocity $v_{\max }$.

The effects of this limitation will be discussed in section 4 In order to 
detect cloud (shadow) movements, the shadow camera needs an reasonably homogeneously area with little non-cloud movements and an elevated position for feasible viewing geometries. In Kuhn et al. (2018b), a system consisting of a shadow camera and an all-sky imager for cloud height determinations is presented. Further applications of shadow cameras are discussed in Kuhn et al. (2017b).

To investigate the cloud motion vectors, each CSS measurement, without any temporal averaging, is compared to the $\pm 2 \mathrm{~min}$ (four-minute) median of the shadow camera measurements. Furthermore, $\pm 2 \min$ (four-minute) and \pm 5 min (ten-minute) medians of the CSS measurements are compared to corresponding shadow camera measurements. If within the individual temporal interval no reference measurement is available, the corresponding CSS measurements are dropped. As the shadow camera approach derives reliably velocities only up to $17.5 \mathrm{~m} / \mathrm{s}$, CSS measurements with a corresponding reference value above this speed are also dropped. For the investigation of cloud motion directions, vectors measured by the CSS and the shadow camera are compared to each other. Without the temporal averaging, the LSQ method is studied on 2956 measurements and the LTS method on 4828 measurements for which shadow camera reference measurements are available. In total, the LSQ method derived 3170 measurements on 59 days, the LTS method 6041 and the shadow camera 23155. To quantify the deviations, root-mean-square deviations (RMSD), meanabsolute deviations (MAD) and the bias are calculated (equ. 7.99 .

$$
\operatorname{RMSD}=\sqrt{\frac{1}{N} \sum_{i=1}^{N}\left(v_{C S S, i}-v_{S C, i}\right)^{2}}
$$

$$
\mathrm{MAD}=\frac{1}{N} \sum_{i=1}^{N}\left|v_{C S S, i}-v_{S C, i}\right|
$$

$$
\text { bias }=\frac{1}{N} \sum_{i=1}^{N}\left(v_{C S S, i}-v_{S C, i}\right)
$$




\section{Benchmarking the CSS}

In section 2.2, an algorithmic change in the software of the CSS is discussed, which significantly increases the amount of detected shading events. In this section, both approaches (LSQ and LTS, see section 2.2) are compared to the shadow camera reference measurements. To begin with, three example days are studied in detail in section 4.1. In section 4.2 cloud shadow speed measurements are studied on 59 days. The directions of the cloud shadows are compared to shadow camera measurements in section 4.3. The detection rate of the CSS is investigated based on its radiometer measurements on 223 days in section 4.4 (not in comparison to the shadow camera). After focussing on the deviations found with the LSQ approach, the deviations of the LTS approach, yielding more measurements, are discussed in section 4.5 .

The speed distributions as measured by the CSS and the shadow camera is depicted in Fig. 7. In the top left, the overall number of occurrence is shown. The shadow camera obtains far more measurements than the CSS, for which the LTS method yields more results than the LSQ method. The vertical line marks the maximum speed reliably resolvable by the shadow camera $(17.5 \mathrm{~m} / \mathrm{s}$, see section 3). This limit was derived for a worst case scenario. Cloud shadows moving diagonally over the imaged area can be reliably measured up to $17.5 \mathrm{~m} / \mathrm{s} \cdot \sqrt{2}=24.7 \mathrm{~m} / \mathrm{s}$. In extreme cases, diagonal cloud shadow speeds up to $525 \mathrm{~m} / 15 \mathrm{~s} \cdot \sqrt{2}=49.5 \mathrm{~m} / \mathrm{s}$ can be measured. However, beyond $17.5 \mathrm{~m} / \mathrm{s}$, the speeds cannot be safely resolved for all directions. $92.6 \%$ of all shadow camera measurements are below $24.7 \mathrm{~m} / \mathrm{s}, 81.4 \%$ of all shadow camera measurements are below $17.5 \mathrm{~m} / \mathrm{s} .92 .1 \%$ of all CSS measurements obtained with the LSQ method are below $17.5 \mathrm{~m} / \mathrm{s}(98.5 \%$ below $24.7 \mathrm{~m} / \mathrm{s}) .93 .0 \%$ of all CSS measurements derived with the LTS method are below $17.5 \mathrm{~m} / \mathrm{s}(98.1 \%$ below $24.7 \mathrm{~m} / \mathrm{s}$ ). Given the distribution of the speeds measured by the CSS and the limitations of the shadow camera, all shadow camera measurements beyond $17.5 \mathrm{~m} / \mathrm{s}$ are excluded from the comparisons in this section. For speeds considered in the following comparisons $(v<=17.5 \mathrm{~m} / \mathrm{s})$, the mean speed of 

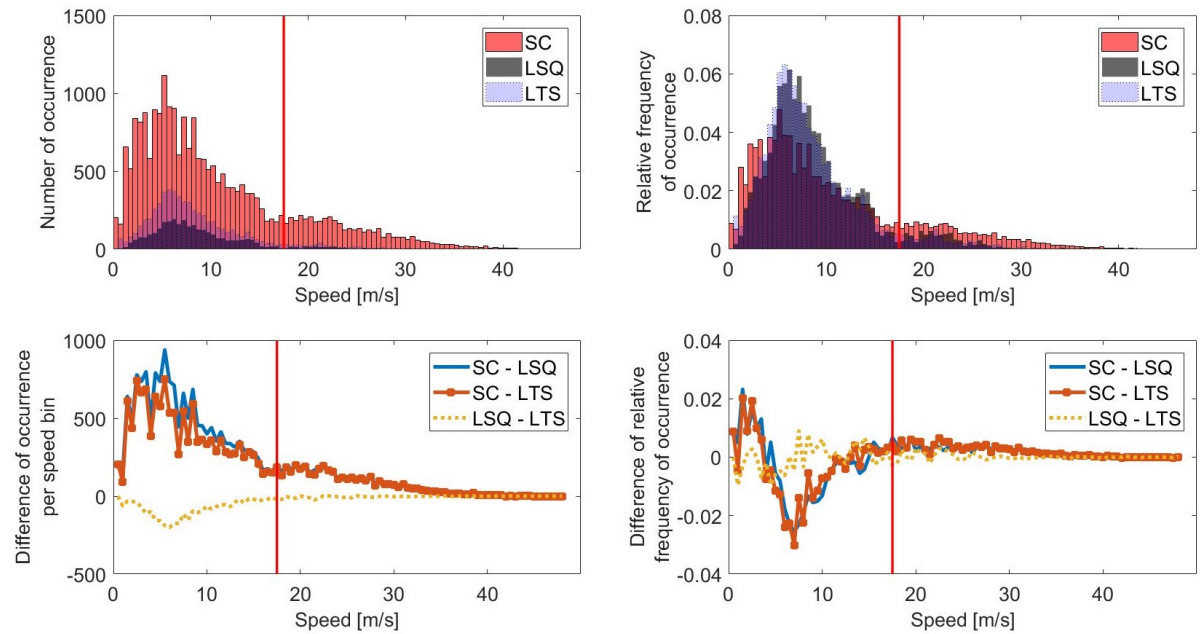

Figure 7: Top left: histograms of all cloud motion vectors obtained on 59 days by the shadow camera (SC), the CSS using the LSQ method (LSQ) and the CSS using the LTS method (LTS). Top right: relative frequency of occurence. Bottom left: bin-wise subtraction of the number of occurrence (see top left). Bottom right: bin-wise subtraction of the relative frequency of occurrence (see top right). The vertical line marks the maximum speed reliably resolvable by the shadow camera for all cloud motion directions.

the shadow camera measurements is $7.36 \mathrm{~m} / \mathrm{s}$ (median: $6.67 \mathrm{~m} / \mathrm{s}$ ), the mean speed of the CSS measurements with the LSQ approach is $8.99 \mathrm{~m} / \mathrm{s}$ (median: $7.69 \mathrm{~m} / \mathrm{s}$ ) and with the LTS approach $8.60 \mathrm{~m} / \mathrm{s}$ (median: $7.30 \mathrm{~m} / \mathrm{s}$ ). Although the modes of the histograms are at $6.0 \mathrm{~m} / \mathrm{s}$, a wide range of cloud speeds are measured.

\subsection{Three example days}

Before looking at long-term comparisons in the next sections, three example days are specifically studied. The example days are 2016-03-19, 2016-04-22 and 2016-10-14. For these example days, the CSS data are shown without any temporal averaging. The effects of temporal averaging on the comparisons are studied in the next sections.

The cloud speeds and direction of 2016-10-14 are shown in Fig. 9. Cloud motion directions are displayed in the top part, cloud velocities in the bottom part. 


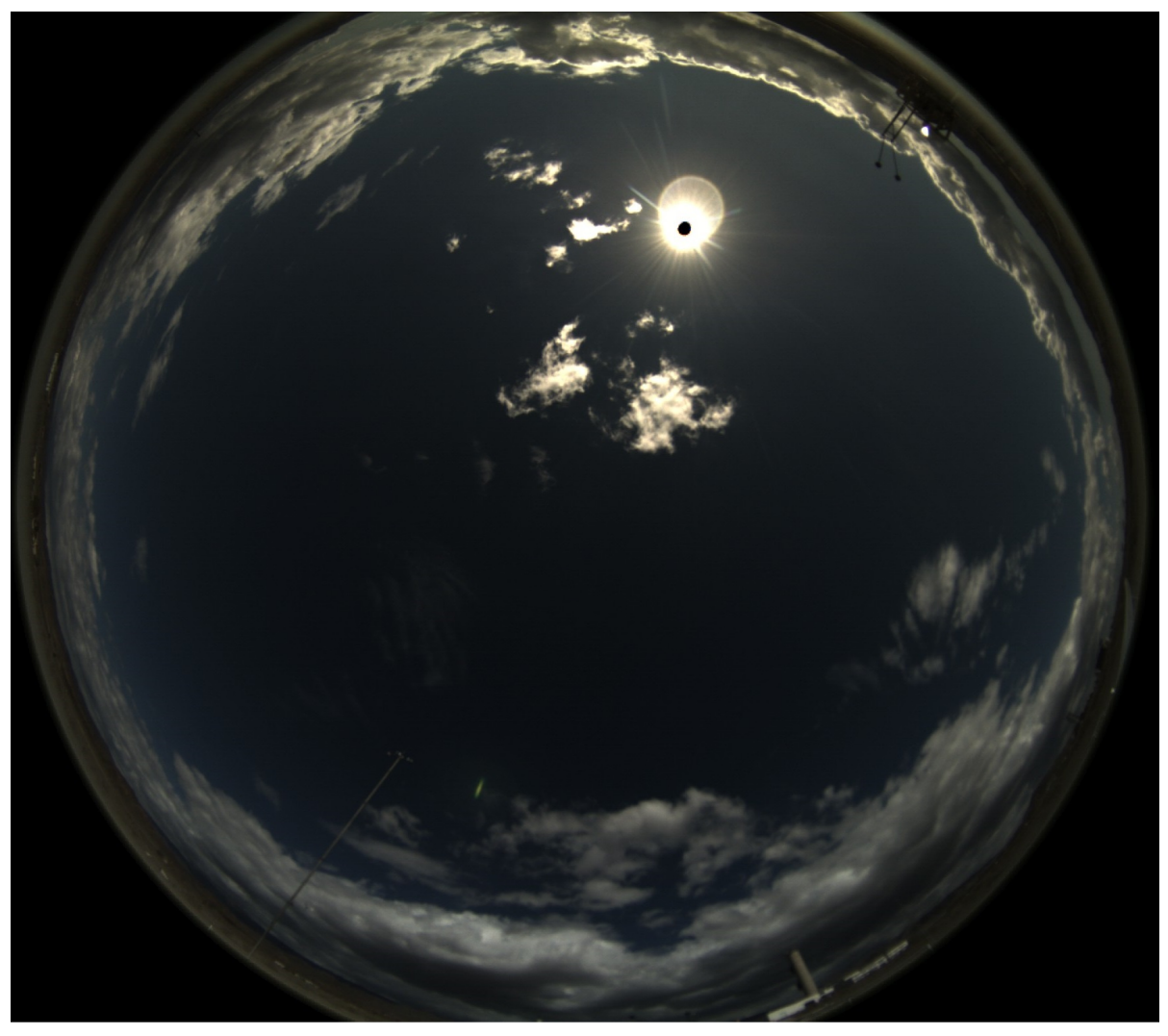

Figure 8: All-sky image taken at 2016-10-14, 12:10:00 UTC+1. Small clouds are visible around the sun, which are dynamically forming.

The values of the reference system are depicted as \pm 2 min medians; the CSS measurements are not additionally averaged or filtered. On this day, altocumulus clouds between 2000 and $3000 \mathrm{~m}$ are predominant, traveling from north-west to south-east. The shadow camera obtained 653 measurements on this day, the CSS with the LSQ method 60 and with the LTS method 111 measurements.

Prior to $12: 31 \mathrm{~h}(\mathrm{UTC}+1)$, the shadow camera does not provide measurements. Looking at the shadow camera video of this day, the lack of measurements can be explained by a lack of (visible) shading events. The shading events measured by the CSS are not visible in the shadow camera video. However, the data of a near-by all-sky imager show that around 12:15 h there are some tiny 

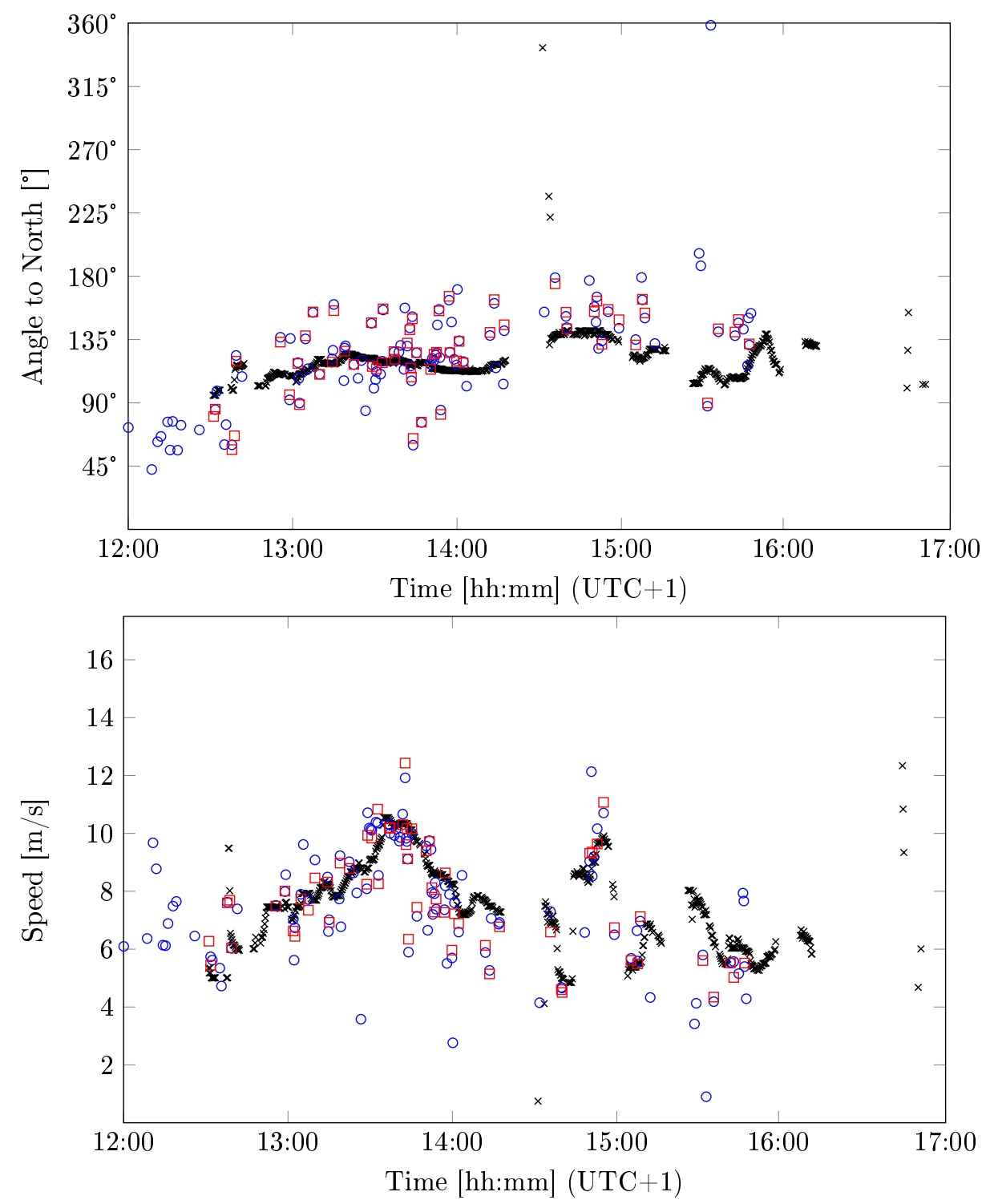

$\times$ Shadow camera
$\circ$ CSS (LTS method)
$\square$ CSS (LSQ method)

Figure 9: CSS and shadow camera measurements on 2016-10-14. The shadow camera reference measurements show less scatter than the CSS measurements. 


\section{in Tab. 1}

Table 1: Deviations between the LSQ and LTS approach in comparison to the shadow camera on 2016-10-14. Instantaneous CSS measurements without any temporal averaging are compared to \pm 2 min medians derived from the shadow camera. The deviations of the cloud motion direction are calculated from vectors.

\begin{tabular}{l|cc} 
& LSQ approach & LTS approach \\
\hline RMSD & $1.1 \mathrm{~m} / \mathrm{s}, 25.6^{\circ}$ & $1.6 \mathrm{~m} / \mathrm{s}, 28.4^{\circ}$ \\
MAD & $0.8 \mathrm{~m} / \mathrm{s}, 20.3^{\circ}$ & $1.1 \mathrm{~m} / \mathrm{s}, 21.0^{\circ}$ \\
bias & $-0.2 \mathrm{~m} / \mathrm{s}, 8.3^{\circ}$ & $-0.4 \mathrm{~m} / \mathrm{s}, 10.1^{\circ}$
\end{tabular}

clouds dynamically forming around the sun (see Fig. 8). This might be an example of a nugget effect with the spatial resolution of the CSS being far higher than the spatial resolution of the shadow camera at the position of the CSS. This effect and its impact on these comparisons are discussed later and partially

Between 12:30 $\mathrm{h}(\mathrm{UTC}+1)$ and 14:30 $\mathrm{h}$, the measured velocities increase from approximately $5 \mathrm{~m} / \mathrm{s}$ to $10 \mathrm{~m} / \mathrm{s}$ and decrease back to approximately $6 \mathrm{~m} / \mathrm{s}$. Later that day, large scattered clouds with different velocities are present. For this day, the CSS measurements and the reference system align very well. Ceilometer data and all-sky imager videos show that there is only one cloud layer present. The deviation found on this day for the LSQ and the LTS method are displayed
66

7

(1)

Figure 10 visualizes cloud shadow speeds on 2016-03-19 as measured by the shadow camera and calculated by the two algorithmic approaches derived from CSS measurements.

There is one dominant cloud direction (from west to east) throughout the day, both for the shadow camera and the CSS. However, there is variation in cloud speed due to clouds at different heights, as suggested by ceilometer and all-sky imager data (not shown). In general, there is much scatter and large deviations between the measurements. This is partially caused by multiple cloud layers present on this day, which pose a challenge both for the shadow camera and the CSS. Moreover, for the CSS, optically thin clouds are challenging. Their 

this day for the LSQ and the LTS method are displayed in Tab. 2.

Table 2: Deviations found for the LSQ and LTS approach in comparison to the shadow camera on 2016-03-19. Instantaneous CSS measurements without any temporal averaging are compared to \pm 2 min medians derived from the shadow camera.

\begin{tabular}{l|cc} 
& LSQ approach & LTS approach \\
\hline RMSD & $2.7 \mathrm{~m} / \mathrm{s}, 31.4^{\circ}$ & $3.9 \mathrm{~m} / \mathrm{s}, 39.5^{\circ}$ \\
MAD & $1.8 \mathrm{~m} / \mathrm{s}, 23.1^{\circ}$ & $2.7 \mathrm{~m} / \mathrm{s}, 29.9^{\circ}$ \\
bias & $-0.7 \mathrm{~m} / \mathrm{s}, 8.3^{\circ}$ & $-1.6 \mathrm{~m} / \mathrm{s}, 9.5^{\circ}$
\end{tabular}



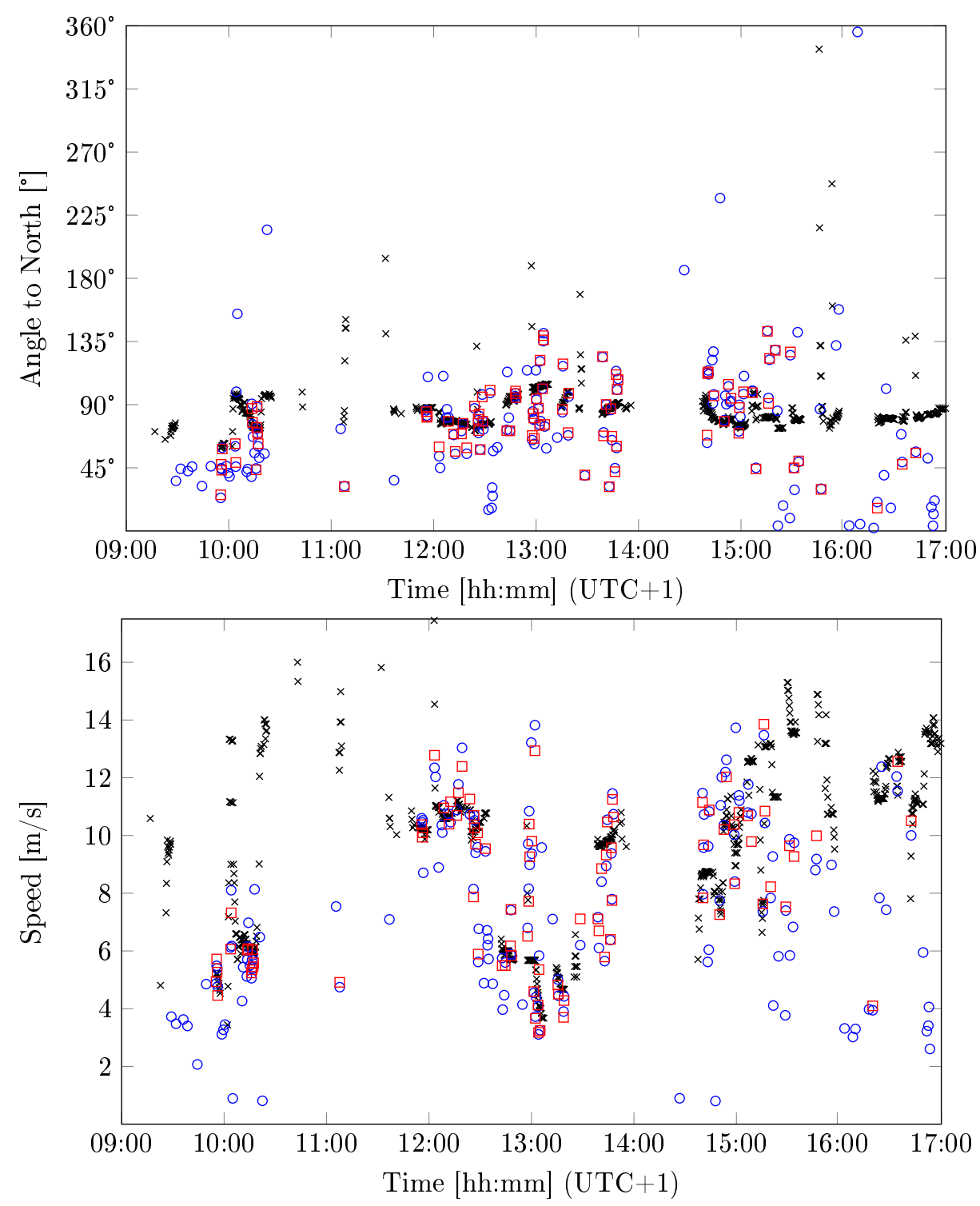

$$
\begin{aligned}
& \times \text { Shadow camera } \\
& \circ \text { CSS (LTS method) } \\
& \square \text { CSS (LSQ method) }
\end{aligned}
$$

Figure 10: CSS and shadow camera measurements on 2016-03-19. Due to multiple cloud layers and optically thin clouds, both scatter and significant deviations between the CSS measurements and the shadow camera reference systems are present. 

on this day for the LSQ and the LTS method are displayed in Tab. 3.

Table 3: Deviations found for the LSQ and LTS approach in comparison to the shadow camera on 2016-04-22. Instantaneous CSS measurements without any temporal averaging are

compared to \pm 2 min medians derived from the shadow camera.

\begin{tabular}{l|cc} 
& LSQ approach & LTS approach \\
\hline RMSD & $1.6 \mathrm{~m} / \mathrm{s}, 24.9^{\circ}$ & $1.9 \mathrm{~m} / \mathrm{s}, 37.8^{\circ}$ \\
MAD & $1.2 \mathrm{~m} / \mathrm{s}, 20.1^{\circ}$ & $1.4 \mathrm{~m} / \mathrm{s}, 25.6^{\circ}$ \\
bias & $-0.8 \mathrm{~m} / \mathrm{s}, 3.9^{\circ}$ & $-0.8 \mathrm{~m} / \mathrm{s}, 1.3^{\circ}$
\end{tabular}
this day, mainly altocumulus clouds with an altitude of $2000 \mathrm{~m}$ are present. Both the measured cloud directions and the measured cloud speeds are not homogeneous throughout the day. Between 11:00 h $(\mathrm{UTC}+1)$ and 12:30 $\mathrm{h}$, the CSS measurements scatter strongly in comparison to the reference system. Also, a bias in the velocities is found. The origins of these deviations lay in a key assumption of the linear cloud edge - curve fitting method, which is discussed in appendix A. Between 13:00 $\mathrm{h}(\mathrm{UTC}+1)$ and 15:00 $\mathrm{h}$, there is a high correlation between the measurements.

Between 16:00 $\mathrm{h}(\mathrm{UTC}+1)$ and 16:30 $\mathrm{h}$, the CSS is shaded by clouds, but does not provide any measurements. Looking at all-sky and shadow camera images as well as ceilometer data reveals that this is caused by optically thin clouds with diffuse edges at approximately $4000 \mathrm{~m}$ altitude. Their speed is beyond the limits of the reference system $(17.5 \mathrm{~m} / \mathrm{s})$.

After $16: 30 \mathrm{~h}(\mathrm{UTC}+1)$, there is a significant amount of scatter. All-sky imager data testify multiple cloud layers during this time. The deviation found

The cloud speeds and direction of 2016-04-22 are depicted in Fig. 11. On 

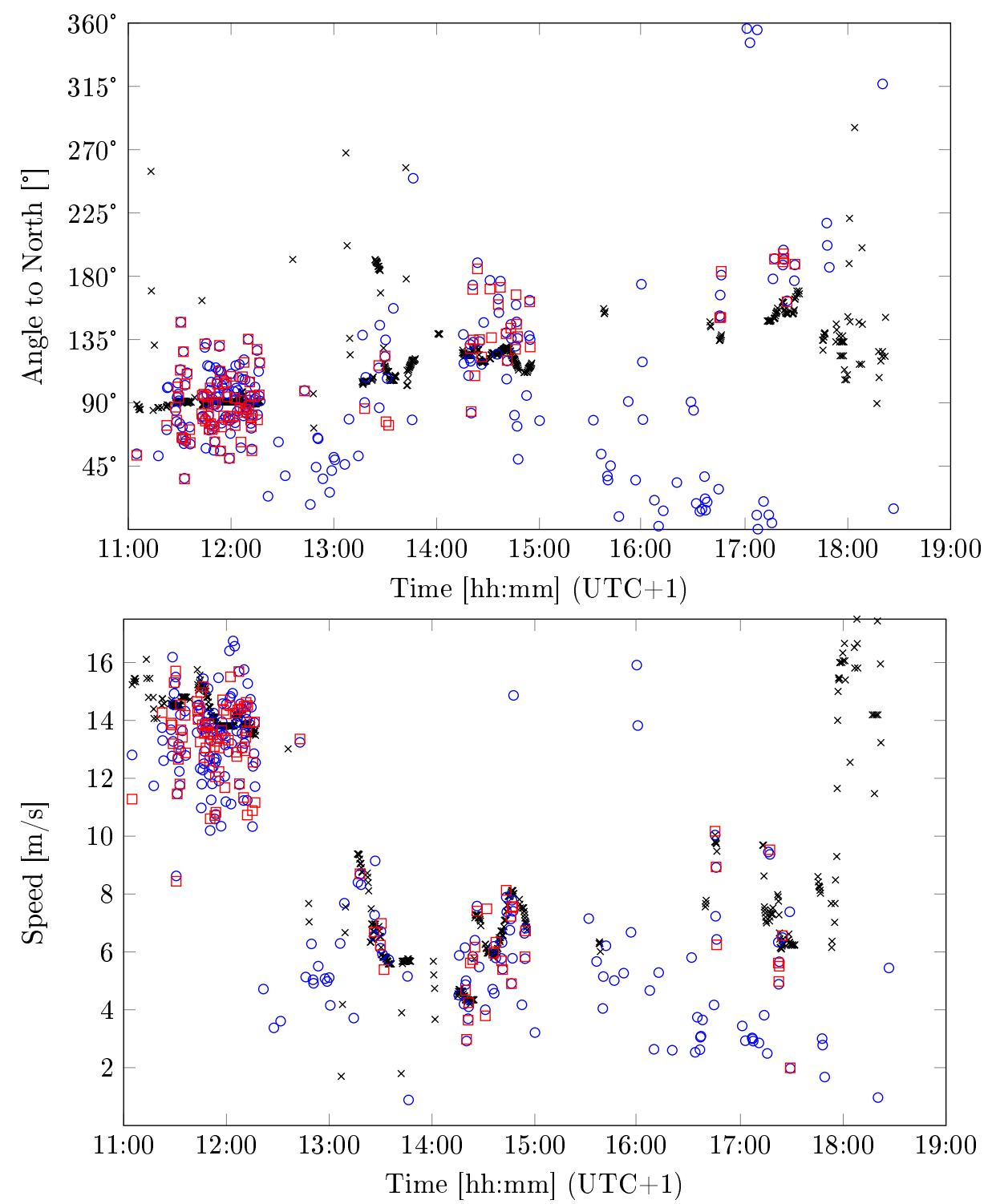

\begin{tabular}{ll|}
$\times$ Shadow camera \\
$\circ$ CSS (LTS method) \\
$\square$ CSS (LSQ method) \\
\hline
\end{tabular}

Figure 11: CSS and shadow camera measurements on 2016-04-22. Both the cloud directions and the cloud speeds change multiple times during the day. 


\subsection{Comparing cloud shadow speeds: CSS against shadow camera}

During the comparison period of 59 days, the CSS obtained 3170 cloud motions vectors with the LSQ approach (for details see section 2.2). The shadow camera measured 23155 cloud motion vectors. This discrepancy between the amount of CSS measurements and the shadow camera approach is partially caused by optically thin clouds, which often do not trigger a CSS measurement (see section 4.4, and by the area of the measurements. The CSS is statistically not shaded as often as the area imaged by the reference system because these two areas have far different sizes (CSS: approximately $0.09 \mathrm{~m}^{2}$; shadow camera: approximately $0.28 \mathrm{~km}^{2}$ ).

The deviations found for the LSQ method in comparison to the shadow camera measurements are displayed in Tab. 4 without any temporal averaging, \pm 2 min medians $\left(\overline{\mathrm{LSQ}}_{ \pm 2 \text { min }}\right)$ and \pm 5 min temporal medians $\left(\overline{\mathrm{LSQ}}_{ \pm 5 \text { min }}\right)$.

The deviations are visualized in a scatter density plot in Fig. 12. The deviations stem mostly from optically thin clouds and clouds at large altitudes (see Kuhn et al. (2018b)). If such clouds trigger CSS measurements at all, the accuracy is poor.

Table 4: Deviations found for the LSQ approach for measurements with and without temporal averaging in comparison to the shadow camera measurements on 59 days (shadow speed).

\begin{tabular}{l|lll} 
& LSQ approach & $\overline{\mathrm{LSQ}}_{ \pm 2} \min$ & $\overline{\mathrm{LSQ}}_{ \pm 5} \min$ \\
\hline RMSD & $2.7 \mathrm{~m} / \mathrm{s}(36.6 \%)$ & $2.4 \mathrm{~m} / \mathrm{s}(32.7 \%)$ & $2.1 \mathrm{~m} / \mathrm{s}(28.0 \%)$ \\
MAD & $1.6 \mathrm{~m} / \mathrm{s}(21.9 \%)$ & $1.3 \mathrm{~m} / \mathrm{s}(18.0 \%)$ & $1.2 \mathrm{~m} / \mathrm{s}(15.7 \%)$ \\
$\operatorname{bias}$ & $-0.2 \mathrm{~m} / \mathrm{s}(2.7 \%)$ & $-0.2 \mathrm{~m} / \mathrm{s}(2.5 \%)$ & $-0.2 \mathrm{~m} / \mathrm{s}(2.8 \%)$
\end{tabular}




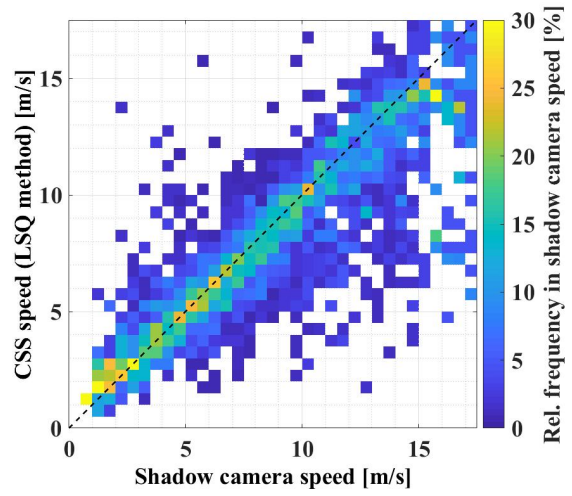

(a)

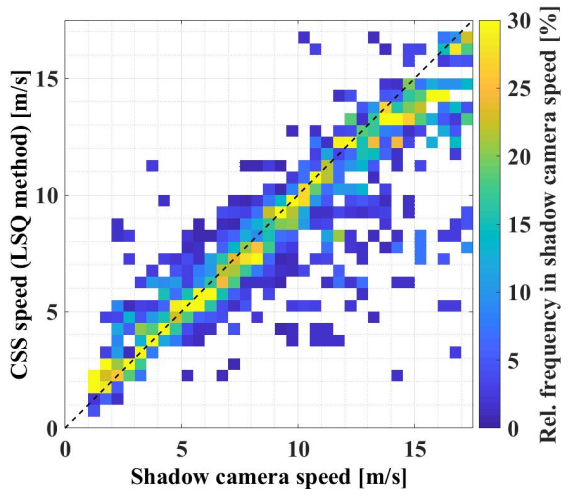

(b)

Figure 12: Scatter density plots of the speeds measured by the CSS and the shadow camera. Figure 12a LSQ method without temporal averaging, Fig. 12b LSQ method with \pm 5 min temporal medians. The colorbar represents the relative frequency of a given pixel within the corresponding shadow camera speed bin. Each column adds up to $100 \%$. In total, the LSQ method obtained 3170 measurements of which 2956 could be temporally matched to shadow camera measurements.

\subsection{Comparing cloud shadow directions: CSS against shadow camera}

This section compares the cloud shadow directions as measured by the CSS against the reference shadow camera. The data set for this comparison is the same as in section 4.2 . The deviations found for the LSQ method in comparison to the shadow camera regarding the shadow directions are displayed in Tab. 5. Although there is only a minor bias present, the deviations do not shrink significantly with larger temporal medians. This is an indication that systematic offsets are present between the CSS and the shadow camera measurements. These offsets can be explained by the different area from which these two systems derive their cloud motion vectors. For the shadow camera, this is a relatively large area. Therefore, the obtained cloud motion direction is an average direction. The CSS, however, might be able to resolve smaller cloud movements, e.g. rotations or very small clouds (such as the clouds at 12:15 h, 2016-10-14, as discussed in section 4.1). Furthermore, the CSS measurements are based on the assumptions of the linear cloud edge - curve fitting method, 
Table 5: Deviations found for the LSQ approach in comparison to the shadow camera approach on 59 days with and without temporal averaging (shadow motion direction, $180^{\circ}=100 \%$ ).

\begin{tabular}{l|lll} 
& LSQ approach & $\overline{\mathrm{LSQ}}_{ \pm 2} \min$ & $\overline{\mathrm{LSQ}}_{ \pm 5 \text { min }}$ \\
\hline RMSD & $50.2^{\circ}(28.0 \%)$ & $52.2^{\circ}(29.0 \%)$ & $47.9^{\circ}(26.6 \%)$ \\
MAD & $30.4^{\circ}(16,8 \%)$ & $28.2^{\circ}(15.6 \%)$ & $25.3^{\circ}(14.0 \%)$ \\
bias & $0.5^{\circ}(0.2 \%)$ & $3.4^{\circ}(2.0 \%)$ & $3.7^{\circ}(2.0 \%)$
\end{tabular}

which is visualized in Fig. 2 and discussed in appendix A. If e.g. a cloud shades the CSS with a saw tooth edge of suitable size, the measured direction might not be the general direction of the cloud. Such systematic offsets could explain the behavior seen in Tab. 5 as well as the scatter seen in Fig. 13 .

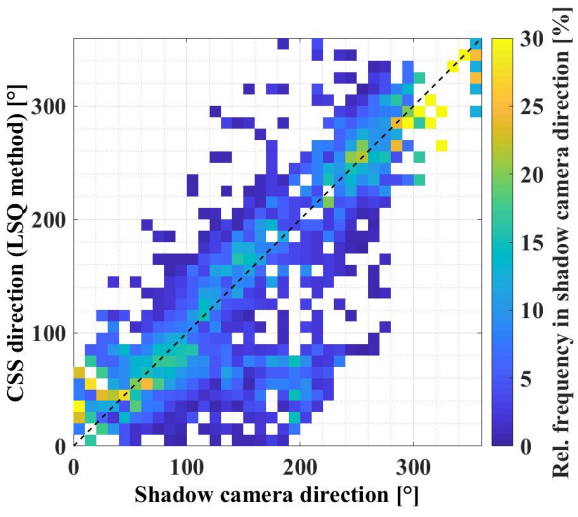

(a)

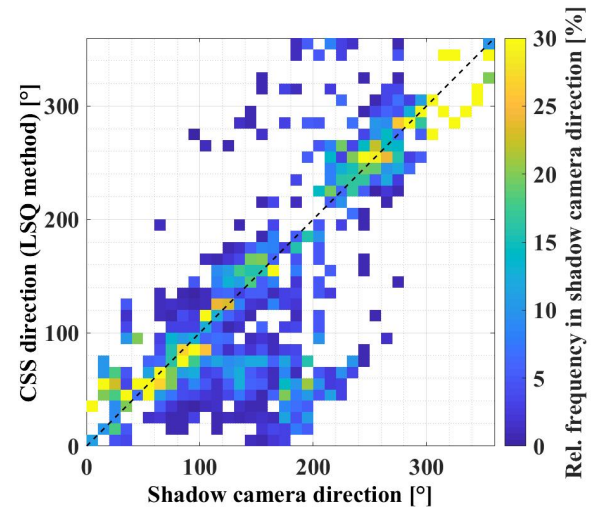

(b)

Figure 13: Scatter density plot of CSS LSQ without temporal averaging (a) and CSS LSQ with \pm 5 min temporal medians (b) cloud directions versus the shadow camera cloud directions. The colorbar represents the relative frequency of a given pixel within the corresponding shadow camera direction bin.

\subsection{Investigating the detection rate of the CSS}

In section 2.2, a method to increase the detection rate of the CSS is discussed.

The validation presented in this section is conducted on 223 days (from 201645 03-20 to 2016-10-28). The validation of the detection rate is not conducted in 
comparison to the shadow camera, but in comparison to normalized irradiance measurements of the CSS itself. This approach is chosen to avoid scale effects between the shadow camera and the CSS. These scale effects are clouds seen by the CSS but not by the shadow camera, clouds imaged by the shadow camera but not shading the CSS and shadows beyond the temporal resolution of one system. The approach to investigate the detection rate of the CSS by looking at the CSS raw data is described in the following.

Figure 14 displays an example day as measured by one of the nine CSS sensors. A clear sky global horizontal irradiance (CSF) model described in Hanrieder et al. (2016) is added and the sensor signals are calibrated to the measurements of a close-by GHI reference station. Furthermore, the $9 \mathrm{~s}$ missing data after each 9 s measurement are linearly interpolated. Using a clear sky

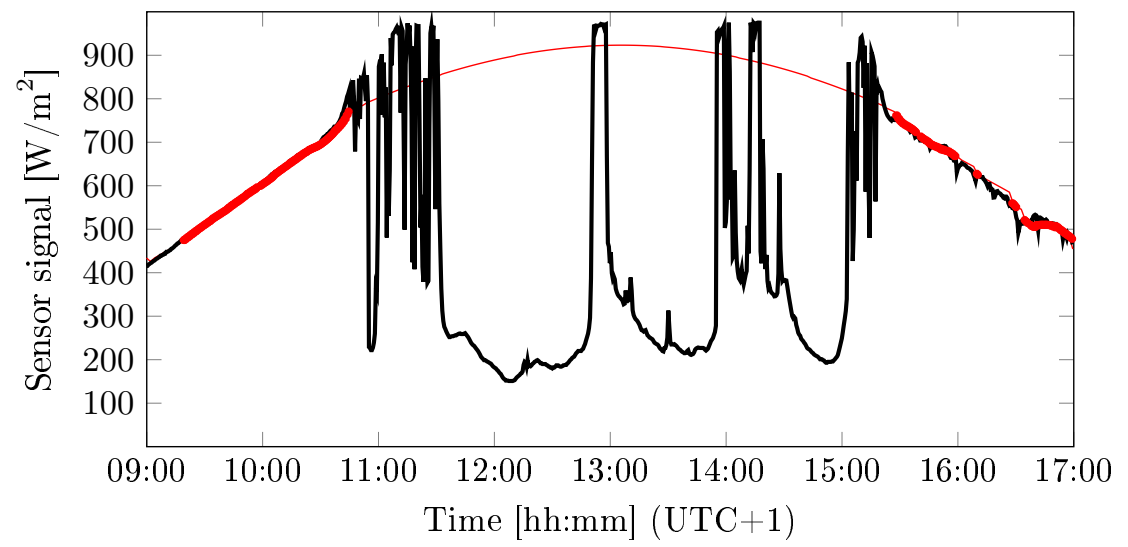

Figure 14: Example day with added clear sky reference (2016-08-25). DHI overshootings and shading events caused by transient clouds are visible.

modeling (CSM), shading strengths (SS) can be defined (Mäki and Valkealahti, 2012):

$$
S S=\frac{G H I^{C S M}-G H I}{G H I^{C S M}}
$$

353 In equation 10, GHI is the measured and calibrated irradiance from one of the $3549 \mathrm{CSS}$ sensors and $G H I^{C S M}$ is the modeled clear sky irradiance. Calibration is performed using another calibrated reference pyranometer approximately $500 \mathrm{~m}$ 
356

away from the CSS and a dynamic adaption factor for the CSS sensor signal. The deviations from the modeled clear sky irradiance are used to determine the amount of shading events detected by the CSS. A shading event begins after the ratio of the measured GHI and the clear sky GHI falls below $90 \%$ and ends if it is again above this threshold. The shading strength is derived from the minimum measured GHI between these two timestamps.

All shadings are characterized into 12 classes by their shadings strengths and shading duration. Shading strengths are divided into three different classes:

- $\leq 30 \%$ for optically thinner clouds

- $>30 \%$ and $\leq 60 \%$ for thicker thin clouds

- > $60 \%$ for optically thicker clouds

Shading durations are resolved into four classes:

$-\leq 60 \mathrm{~s}$ for short shading durations

- $>60 \mathrm{~s}$ and $\leq 300 \mathrm{~s}$ for medium shading durations

- $>300 \mathrm{~s}$ and $\leq 600 \mathrm{~s}$ for long shading durations

- $>600 \mathrm{~s}$ for (partial) overcast situations

The relative share of each class as measured from 2016-03-20 to 2016-10-28 (223 days) is shown in Tab. 6. Predominantly, there are optically thin clouds with short shading durations above the PSA. 
Table 6: Classifications based on shading strength and shading duration: Amount of events per class from 2016-03-20 to 2016-10-28 (223 days). Optically thin clouds with short shading durations are most common. Total amount of shading events (per sensor): 8276 .

\begin{tabular}{|c|c|c|c|c|c|c|}
\hline & & \multicolumn{4}{|c|}{ Shading duration $[\mathrm{s}]$} & \multirow[b]{2}{*}{ sum } \\
\hline & & $<60$ & $60-300$ & $300-600$ & $>600$ & \\
\hline \multirow{4}{*}{$\begin{array}{l}\text { Shading } \\
\text { strengh }\end{array}$} & $>60 \%$ & $3.4 \%$ & $3.8 \%$ & $0.9 \%$ & $2.4 \%$ & $10.5 \%$ \\
\hline & $30-60 \%$ & $18.3 \%$ & $8.4 \%$ & $1.8 \%$ & $1.9 \%$ & $30.4 \%$ \\
\hline & $<\mathbf{3 0} \%$ & $52.9 \%$ & $5.3 \%$ & $0.7 \%$ & $0.3 \%$ & $59.1 \%$ \\
\hline & sum & $74.6 \%$ & $17.4 \%$ & $3.4 \%$ & $4.6 \%$ & \\
\hline
\end{tabular}

In Tab. 7, the detected CSS measurements per shading class are depicted using the LSQ approach. The CSS measures only $4.8 \%$ of optically thin clouds with shading durations above $600 \mathrm{~s}$ and is best for optically thick clouds with short shading durations (21.6\% detected events). The rate of successfully detected shading events is low.

Using the LSQ approach (see section 2.2 5830 shading events are detected between 2016-03-20 and 2016-10-28 ( 223 days).

Table 7: Detection rates for each shading class: Relative share of shading events detected by the CSS using the LSQ algorithm from 2016-03-20 to 2016-10-28 (223 days). Total amount of detected shading events: 8276 .

\begin{tabular}{lrrrcc}
\hline & & \multicolumn{4}{c}{ Shading duration [s] } \\
& & \multicolumn{6}{c}{$\mathbf{6 0}$} & $\mathbf{6 0}-\mathbf{3 0 0}$ & $\mathbf{3 0 0}-\mathbf{6 0 0}$ & $>\mathbf{6 0 0}$ \\
\hline \multirow{2}{*}{ Shading } & $>\mathbf{6 0} \%$ & $21.6 \%$ & $16.4 \%$ & $16.7 \%$ & $9.5 \%$ \\
strength & $\mathbf{3 0}-\mathbf{6 0} \%$ & $16.0 \%$ & $13.7 \%$ & $9.5 \%$ & $6.3 \%$ \\
& $<\mathbf{3 0 \%}$ & $8.0 \%$ & $3.7 \%$ & $3.7 \%$ & $4.8 \%$ \\
\hline
\end{tabular}




\subsection{Comparing CSS software approaches: LSQ and LTS}

In section 2.2 , the methodology used by the CSS to derive cloud motion vectors is presented and ways to increase the dectection rate are discussed. As can be seen in section 4.4 the detection rate is low. This can be improved by using the LTS approach instead of the LSQ approach. In this section, the deviations found in comparison to the shadow camera using the CSS with the LTS approach are investigated. Moreover, these deviations are compared to the deviations obtained with the CSS and the LSQ approach.

In comparison to the histogram found for the LSQ approach (see Fig. 7), no significant deviations are present. During the comparison period of 59 days, the CSS obtained 6041 cloud motion vectors using the LTS method (3170 for the LSQ approach, 23155 with the shadow camera).

The deviations found for the LSQ and LTS method in comparison to the shadow camera measurements are displayed in Tab. 8 without any temporal averaging, \pm 2 min medians and \pm 5 min medians. The LTS approach shows higher deviations in comparison to the shadow camera. However, for $\pm 5 \mathrm{~min}$ temporal medians (LSQ: 2705 temporally averaged measurements with corresponding shadow camera reference measurements, LTS: 4350 measurements), the deviations for both LSQ and LTS are similar.

In general, the measurements obtained by the LTS method are less accurate, but far more frequent in comparison to the LSQ method. This is also visualized in the scatter density plots in Fig. 15 .

Table 9 investigates the origin of the larger deviations found using the LTS method. LTS $\in$ LSQ derives the deviations for all LTS measurements which are

Table 8: Deviations found for the LSQ and LTS approach for measurements with and without temporal averaging in comparison to the shadow camera measurements on 59 days (shadow speed).

\begin{tabular}{l|llllll} 
& LSQ approach & $\overline{\mathrm{LSQ}}_{ \pm 2 \mathrm{~min}}$ & $\overline{\mathrm{LSQ}}_{ \pm 5 \mathrm{~min}}$ & LTS approach & $\overline{\mathrm{LTS}}_{ \pm 2 \mathrm{~min}}$ & $\overline{\mathrm{LTS}}_{ \pm 5 \mathrm{~min}}$ \\
\hline RMSD & $2.7 \mathrm{~m} / \mathrm{s}(36.6 \%)$ & $2.4 \mathrm{~m} / \mathrm{s}(32.7 \%)$ & $2.1 \mathrm{~m} / \mathrm{s}(28.0 \%)$ & $3.4 \mathrm{~m} / \mathrm{s}(45.8 \%)$ & $2.9 \mathrm{~m} / \mathrm{s}(39.2 \%)$ & $2.6 \mathrm{~m} / \mathrm{s}(35.2 \%)$ \\
MAD & $1.6 \mathrm{~m} / \mathrm{s}(21.9 \%)$ & $1.3 \mathrm{~m} / \mathrm{s}(18.0 \%)$ & $1.2 \mathrm{~m} / \mathrm{s}(15.7 \%)$ & $2.1 \mathrm{~m} / \mathrm{s}(28.0 \%)$ & $1.7 \mathrm{~m} / \mathrm{s}(22.4 \%)$ & $1.5 \mathrm{~m} / \mathrm{s}(20.2 \%)$ \\
bias & $-0.2 \mathrm{~m} / \mathrm{s}(-2.7 \%)$ & $-0.2 \mathrm{~m} / \mathrm{s}(-2.5 \%)$ & $-0.2 \mathrm{~m} / \mathrm{s}(-2.8 \%)$ & $-0.4 \mathrm{~m} / \mathrm{s}(-5.8 \%)$ & $-0.4 \mathrm{~m} / \mathrm{s}(-5.1 \%)$ & $-0.4 \mathrm{~m} / \mathrm{s}(-5.7 \%)$
\end{tabular}


Table 9: Deviations found for LTS approach adjacent and not adjacent to obtained LSQ measurements in comparison to the shadow camera measurements on 59 days (shadow speed).

\begin{tabular}{|c|c|c|c|c|}
\hline & $\mathrm{LTS} \in \mathrm{LSQ}$ & ${\overline{\mathrm{LTS}} \in \mathrm{LSQ}_{ \pm 1 \mathrm{~min}}}$ & LTS $\notin \mathrm{LSQ}$ & ${\overline{\mathrm{LTS}} \notin \mathrm{LSQ}_{ \pm 1 \mathrm{~min}}}$ \\
\hline RMSD & $2.9 \mathrm{~m} / \mathrm{s}(39.0 \%)$ & $2.4 \mathrm{~m} / \mathrm{s}(32.0 \%)$ & $5.4 \mathrm{~m} / \mathrm{s}(73.2 \%)$ & $5.2 \mathrm{~m} / \mathrm{s}(70.6 \%)$ \\
\hline MAD & $1.8 \mathrm{~m} / \mathrm{s}(24.2 \%)$ & $1.4 \mathrm{~m} / \mathrm{s}(19.3 \%)$ & $3.7 \mathrm{~m} / \mathrm{s}(49.7 \%)$ & $3.5 \mathrm{~m} / \mathrm{s}(47.2 \%)$ \\
\hline bias & $-0.2 \mathrm{~m} / \mathrm{s}(-3.0 \%)$ & $-0.2 \mathrm{~m} / \mathrm{s}(-2.7 \%)$ & $-1.6 \mathrm{~m} / \mathrm{s}(-21.2 \%)$ & $-1.6 \mathrm{~m} / \mathrm{s}(-21.8 \%)$ \\
\hline
\end{tabular}

406

within \pm 1 min around a LSQ measurement $(3517,84.8 \%) .{\overline{\mathrm{LTS}} \in \mathrm{LSQ}_{2} \min }_{1}$ compares these \pm 1 min temporal medians to the shadow camera measurements. LTS $\notin$ LSQ calculates the deviations for LTS measurements, which are not within \pm 1 min around a LSQ measurement $(630,15.2 \%) .{\overline{\operatorname{LTS}} \notin \mathrm{LSQ}_{2} \text { min }}_{2}$ derives the deviations for these measurements as medians over \pm 1 min.

The measurements rejected by the LSQ approach but accepted by the LTS method show far higher deviations in comparison to the shadow camera measurements. Thus the LTS method, providing more measurements, shows similar deviations for situations in which the LSQ method obtains measurements but displays high deviations otherwise.

Figure $15 \mathrm{~b}$ compares the velocities derived from the LSQ and LTS method to each other by taking the \pm 2 min median of the LSQ measurements around a LTS measurement. No systematic bias is present and there is a high correlation. The largest deviations occur for velocities above $15 \mathrm{~m} / \mathrm{s}$. 


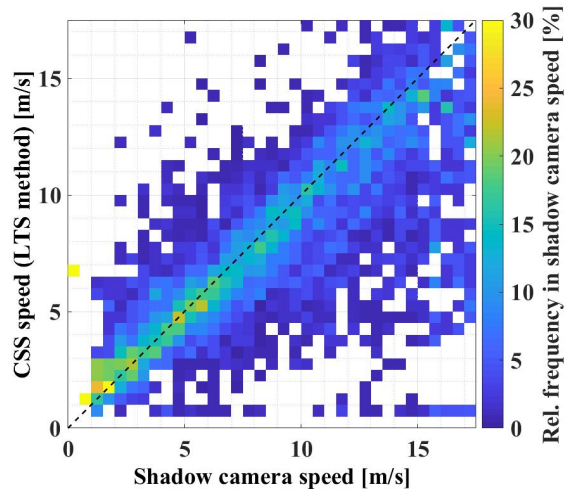

(a)

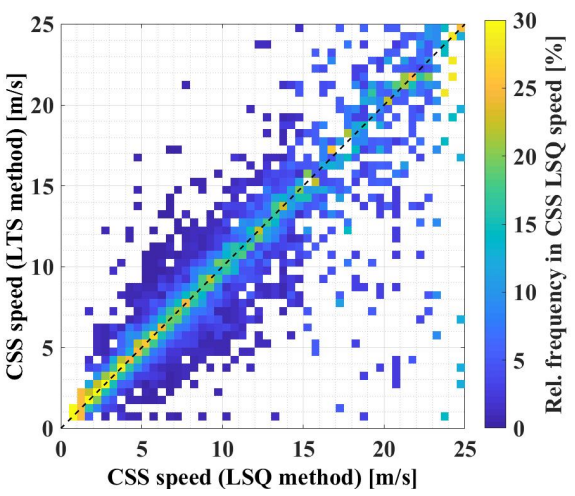

(b)

Figure 15: Scatter density plots of measured cloud speeds on 59 days. Figure 15a LTS method (no temporal averaging, compare to Fig. 12, Fig. 15b. LSQ-LTS comparison. The colorbar represents the relative frequency of a given pixel within the corresponding shadow camera speed bin. Each column adds up to $100 \%$. In total, with the LSQ and LTS method, 3170 and 6041 measurements could be obtained, respectively. The shadow camera produced 23155 measurements.

The deviations found for the LSQ and LTS method in comparison to the shadow camera regarding the shadow directions are displayed in Tab.4.5. Similar to the deviations found for the velocities, the deviations for the LTS method are larger. However, more measurements are obtained with the LTS method in comparison to the LSQ method. As discussed for the direction deviations derived with the LSQ method (see section 4.3), temporal averaging does not reduce deviations as strongly as for the cloud velocities (compare with Tab. 8).

Table 10: Deviations found for the LSQ and LTS approach in comparison to the shadow camera approach on 59 days with and without temporal averaging (shadow motion direction, $180^{\circ}=100 \%$ ).

\begin{tabular}{l|llllll} 
& LSQ approach & $\overline{\mathrm{LSQ}}_{ \pm 2 \text { min }}$ & $\overline{\mathrm{LSQ}}_{ \pm 5 \text { min }}$ & LTS approach & $\overline{\mathrm{LTS}}_{ \pm 2 \text { min }}$ & $\overline{\mathrm{LTS}}_{ \pm 5 \text { min }}$ \\
\hline RMSD & $50.2^{\circ}(28.0 \%)$ & $52.2^{\circ}(29.0 \%)$ & $47.9^{\circ}(26.6 \%)$ & $58.4^{\circ}(32.4 \%)$ & $56.0^{\circ}(30.8 \%)$ & $55.2^{\circ}(30.6 \%)$ \\
MAD & $30.4^{\circ}(16,8 \%)$ & $28.2^{\circ}(15.6 \%)$ & $25.3^{\circ}(14.0 \%)$ & $35.7^{\circ}(20.0 \%)$ & $30.8^{\circ}(17.2 \%)$ & $30.0^{\circ}(16.4 \%)$ \\
bias & $0.5^{\circ}(0.2 \%)$ & $3.4^{\circ}(2.0 \%)$ & $3.7^{\circ}(2.0 \%)$ & $1.1^{\circ}(0.6 \%)$ & $3.0^{\circ}(1.6 \%)$ & $4.4^{\circ}(2.4 \%)$
\end{tabular}


In Fig. 16, the LTS derived cloud shadow directions without temporal averaging are compared to corresponding shadow camera measurements and measurements obtained from the CSS-LSQ approach. Although the measurements align, there is a significant amount of scatter.

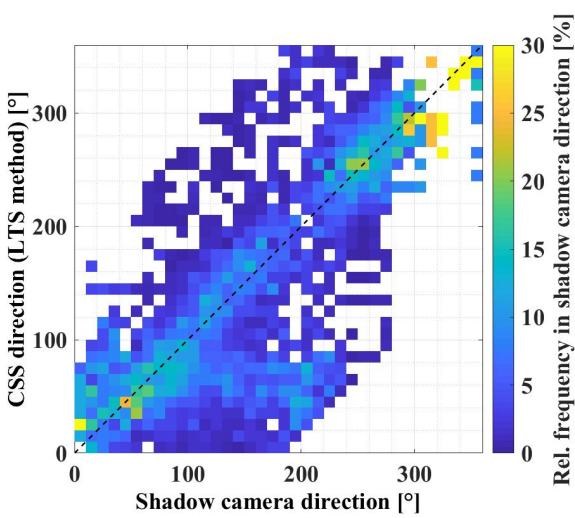

(a)

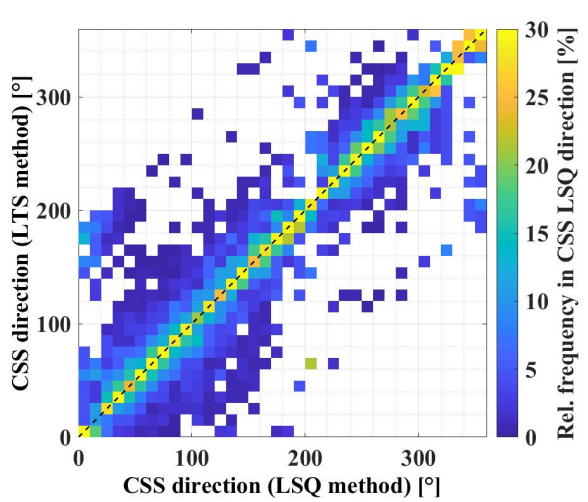

(b)

Figure 16: Scatter density plot of CSS LTS cloud directions without temporal medians versus the shadow camera cloud directions (a) and versus CSS LSQ cloud directions (b), both with temporal medians of \pm 2 min.

Figure 16b compares the directions obtained from the CSS with the LSQ and LTS method using a scatter density plot. The approach is similar to the approach for Fig. 15b. Although there is scatter, the two methods provide similar cloud directions for temporally adjacent measurements (see Tab. 9).

As a conclusion, the LTS method obtains more measurements than the LSQ method. However, for LTS measurements not temporally adjacent to LSQ measurements, the deviations in comparison to the shadow camera are large. However, for some applications (e.g. industrially used cloud height measurement systems) a less accurate measurement might be better than no measurement at all and the LTS method can provide this trade-off. 


\section{Caveats, advantages and disadvantages of the CSS and the novel shadow camera approach}

The shadow camera needs proper orientation, an elevated position and an area with little non-cloud movements. Also, pixels imaging mirrors and other reflective objects cannot be evaluated. Furthermore, evaluating pixels imaging photovoltaic panels or larger vegetation (e.g. forests) is difficult. Although the lack of a strongly elevated position can be overcome by using elevated structures of lower height (e.g. $10 \mathrm{~m}$ ) and a higher image acquisition frequency, such a system would have a disadvantage due to the smaller imaged area. If needed, this issue could be overcome using multiple cameras.

One major disadvantage of this particular shadow camera is the temporal availability of historic images. If an image is taken only every $15 \mathrm{~s}$, very fast clouds will already have transitioned past the image area. Changing the temporal resolution to multiple images per second requires only a simple software adjustment in the camera, but the data storage requirements become prohibitive. For instance, a camera taking $3 \mathrm{MP}$ images every $15 \mathrm{~s}$ accumulates on one day over $12 \mathrm{~h}$ approximately $0.7 \mathrm{~GB}$ of data (255.5 GB per year). An image acquisition rate of $1 \mathrm{~s}$ would increase this figure to approximately $10.4 \mathrm{~GB}$ per day (3.8 TB per year). If 25 images are taken every second, one 3 MP camera produces approximately $259 \mathrm{~GB}$ of data during $12 \mathrm{~h}$ (94.5 TB per year).

If only real-time cloud shadow speeds are of interest, the maximum temporal resolution is just limited by the calculation time. The required time to derive cloud motion vectors strongly depends on the data transmission rate and can in total be below $1 \mathrm{~s}$, which is faster than the calculations of the CSS. With higher temporal resolutions, the area needed to derive (fast) cloud shadow speeds shrinks. However, as many cloud motion vectors should be measured, the imaged area should not be below a certain minimum. This minimum depends on local characteristics and restrictions as well as the intended application.

The CSS however is a fairly compact device, which can be installed at every position which is not shaded by objects. A disadvantage is the detection rate 
and detection accuracy regarding optically thin clouds. As these clouds are less relevant for e.g. photovoltaic nowcasting applications, this might be acceptable.

In direct comparison, the shadow camera obtains more measurements, which scatter less. Also, optically thin clouds can be measured more accurately than with the CSS. Furthermore, the shadow-camera-based approach takes the average cloud motion vector over a larger area, which is more likely to contain cloud shadows than the relatively small area covered by the CSS. Moreover, due to the finite size of cloud shadows, the shadow camera does not face the challenge of the linear cloud edge - curve fitting method as strongly as the CSS (see section A).

In general, both systems require little to no maintenance and were found to be robust in the harsh environments present in the desert of Tabernas. Specifically, the downward-facing shadow cameras require far less maintenance than the upward-facing all-sky imagers.

\section{Conclusion and future work}

On 59 days, the cloud shadow speeds and the cloud directions measured by the CSS are compared to a novel shadow camera approach for two algorithmic methods. For \pm 5 min temporal medians, deviations of RMSD $2.1 \mathrm{~m} / \mathrm{s}(28.0 \%)$, MAD $1.2 \mathrm{~m} / \mathrm{s}(15.7 \%)$ and a bias of $-0.2 \mathrm{~m} / \mathrm{s}(2.8 \%)$ are found. Deviations of the cloud shadow direction are RMSD $47.9^{\circ}$ (26.6\%), MAD $25.3^{\circ}(14.0 \%)$ and a bias $3.7^{\circ}(2.0 \%)$. An alternative algorithm, obtaining more measurements, shows higher deviations. In addition to that, the detection rate of the CSS is determined to be between $3.7 \%$ and $21.6 \%$ depending on the shading class on 223 days.

The effects of the linear cloud edge - curve fitting method are studied and potential solutions discussed. The effects were found to be of minor importance. Potential corrections approaches were found to increase deviations. Thus, we suggest not applying them.

As the CSS and the reference shadow camera can be used for the same 
501

purposes, the specific advantages and disadvantages are discussed. The CSS is found to be the more flexible tool. However, given certain infrastructural / geographical requirements, the shadow camera might be the better choice. Both systems do not require regular maintenance and come with a small price tag (although the CSS is currently not commercially available).

As shown, strict filtering of CSS measurements leads to very little data with many shading events not being measured. If the filtering is less strict, the measurements show larger deviations. Depending on the application, a less accurate measurement might be more desirable than no measurement at all. For instance, if clouds speeds are used to obtain cloud heights for a nowcasting system used in industry, less accurate measurements can be preferable to missing measurements. If on the other hand reference data for validations are to be obtained, accuracy might be more important than the total amount of measurements. Therefore, as a software improvement, we suggest making this decision based on the requirements for each application.

The CSS used in this study measures for $9 \mathrm{~s}$ and stores the results afterwards, which causes a dead time of another $9 \mathrm{~s}$. Although this dead time can be interpolated, continuous measurements would further improve the device. In a redesigned version of the CSS (developed in late 2016), the dead time was reduced to $2 \mathrm{~s}$. Future hardware improvements should further reduce this dead time.

In many cases, cloud shadow speeds are not the final measurement of interest but only an intermediate result. Depending on the intended application of the CSS, several other potential hardware adaptions could be implemented. If irradiance values are of interest, one or several sensors of the CSS could be calibrated and thus used to measure GHI. Integrating a rotating shadow band (RSI) into the CSS would further enable direct normal irradiance (DNI) measurements. If the CSS is used as a part of an all-sky imager based nowcasting system or utilized to derive cloud heights, an inexpensive camera could be added, providing a complete system. A CSS and a shadow camera based system, which derives cloud heights, is presented and validated against a ceilometer on the 
545 camera system (PreFlexMS, Grant Agreement no. 654984). With founding

546 from the German Federal Ministry for Economic Affairs and Energy within

${ }_{547}$ the WobaS project, the shadow camera system was developed. The European

${ }_{548}$ Union's FP7 program under Grant Agreement no. 608623 (DNICast project)

549 financed operations of the all-sky imagers and other ground measurements. The

$5 \mathbf{5 0}$ authors are also grateful for the financial support provided by project PRESOL

551 with reference ENE2014-59454-C3-2-R, funded by the Ministerio de Economía y

552 Competitividad and co-financed by the European Regional Development Fund

553 (FEDER). Thanks to the reviewers for their helpful comments and to our col-

554 leagues from the Solar Concentrating Systems Unit of CIEMAT for the support

555 provided in the installation and maintenance of the shadow cameras. These

556 instruments are installed on CIEMAT's CESA-I tower of the Plataforma Solar

557 de Almería. 


\section{Appendix A Angle correction and the linear cloud edge - curve fitting method}

Here, basic assumptions of the linear cloud edge - curve fitting method are studied and potential solutions discussed. The considerations are not only relevant for the CSS, but for many other velocity deriving systems. These investigations require a reference system. The shadow camera provides such references, enabling us to carry out these studies on the CSS. To the best of our knowledge, this is the first time such an in-field investigation of the aperture problem is performed.

\section{A.1 The aperture problem on one example day}

The aperture problem is a very fundamental challenge for many velocity deriving systems. Several publications on the CSS and on similar systems (Bosch and Kleissl (2013), Bosch et al. (2013), Lappalainen and Valkealahti (2016a),

Lappalainen and Valkealahti (2016b)) use the linear cloud edge method to overcome this problem. In this method, the cloud speed and the moving direction of the cloud are determined from the measurements obtained by two shading flanks with assumed identical cloud motion vectors. To avoid this assumption, the "linear cloud edge - curve fitting method" is implemented in the CSS (Wang et al. 2016). This method assumes that the motion of a cloud is always perpendicular to the cloud edge (see Fig. 1). If the cloud edge is not perpendicular to the moving direction of the cloud, the cloud speed is underestimated by the factor $\cos \delta$, where $\delta$ represents the angle between the speed vector and the normal of the shadow edge. This question has been addressed in previous works but no sufficient answer has been found yet (Bosch et al. (2013), Lappalainen and Valkealahti (2016a)). With the shadow camera acting as a reference, the effects of these systematic deviations can be studied and reversed. Figure A.1 visualizes the raw data of the CSS measurements and the shadow camera measurements for speed and direction for one example day (2016-04-25) without any temporal averaging for both systems. The CSS measurements scatter in a 

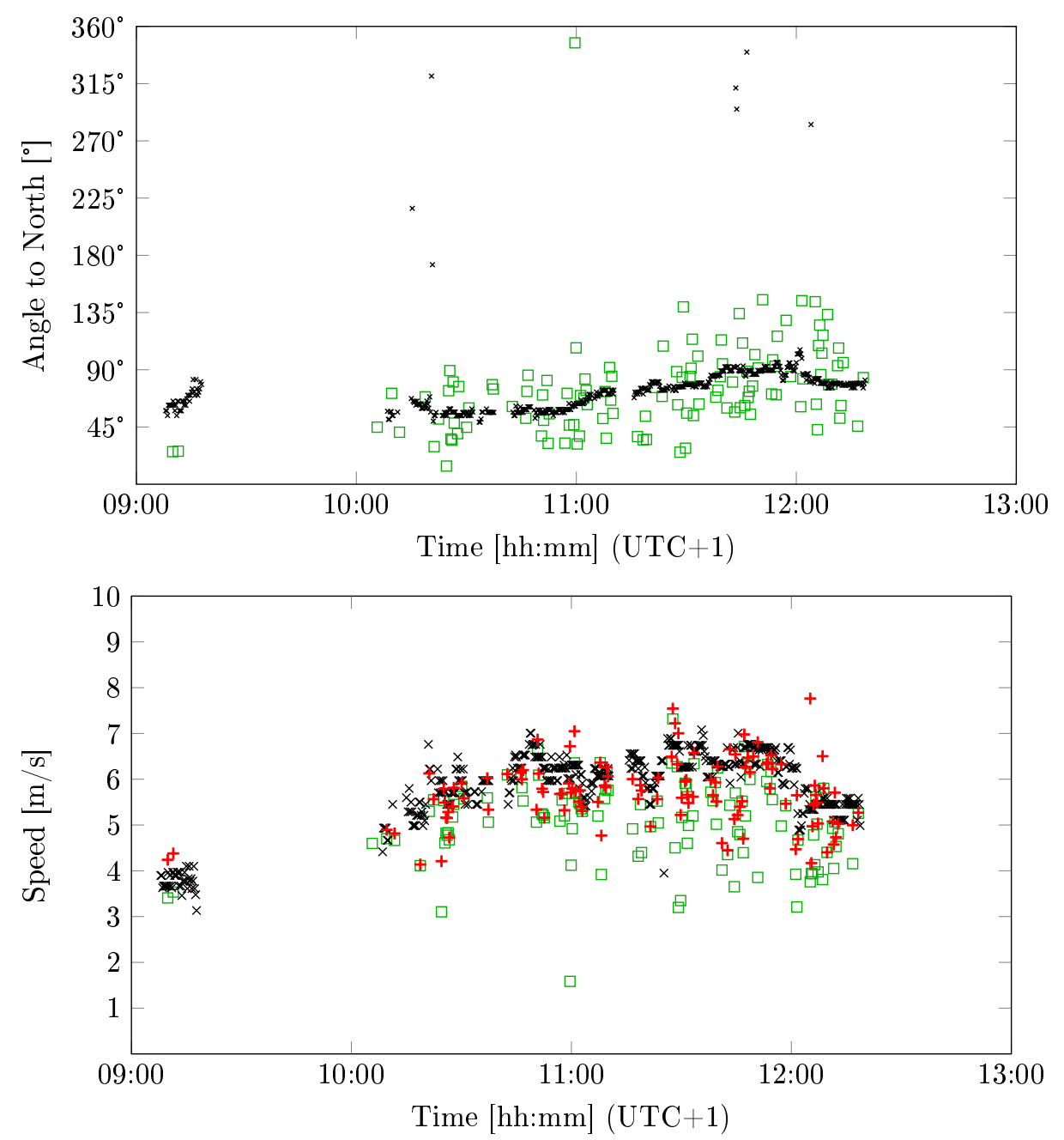

$\square$ Cloud Speed Sensor

$\times$ Shadow camera

+ corrected CSS speed

Figure A.1: CSS measurements and the raw data of the shadow camera on 2016-04-25. This example is used to illustrate the effects of the linear cloud edge method.

587

588

589

590

significant range, whereas the shadow camera system cloud motion directions

show almost no scatter at all and only a minor number of outliers throughout

the day. The low level of scatter and bias in the raw data is a strong indication that the direction detected by the shadow camera is correct. We will show 


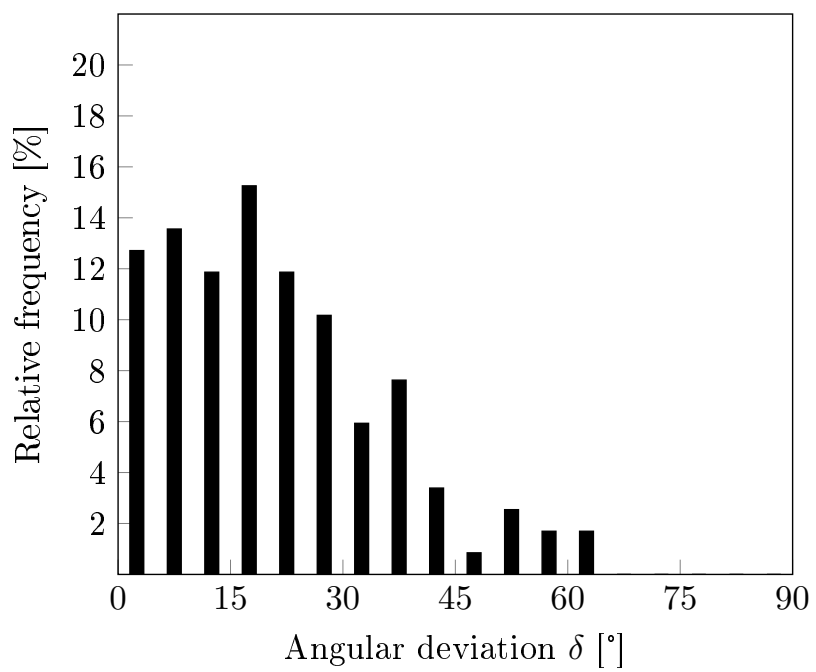

Figure A.2: Angular deviation $\delta$ on 2016-04-25 between the one-shadow-camera system and the CSS, depicted for the LSQ method. There is a total of 118 CSS measurements using the LSQ method.

591 in this section that scatter in the CSS data is partially caused by cloud edges passing the CSS not being perpendicular to the motion vectors.

In the following, the moving direction measured by the shadow camera is considered the true direction of the clouds, which appears justified because its scatter is very small. The distribution of the thus measured angular deviation $\delta$ between the CSS measurements (displayed for the LSQ method) and the reference system is shown in Fig. A.2. The deviations are significant and result in systematically too small speeds as measured by the CSS.

With $\delta$ known, the CSS speed can be corrected according to equ. A.1 (compare with Fig. 2). The corrected CSS velocities are depicted with + in the bottom part of Fig. A.1. Due to the correction, the scatter is reduced from $0.9 \mathrm{~m} / \mathrm{s}$ to $0.7 \mathrm{~m} / \mathrm{s}$ standard deviation. Furthermore, the corrected average speed $(5.7 \mathrm{~m} / \mathrm{s})$ on this day of is closer to the average speed as measured by the shadow camera $(6.2 \mathrm{~m} / \mathrm{s})$ than the uncorrected average speed $(5.1 \mathrm{~m} / \mathrm{s})$.

$$
v_{C S S}^{c o r r}=\frac{v_{C S S}}{\cos \delta}
$$




\section{A.2 Investigating potential solutions}

Assuming that the bias (presented in section 4.5 is only caused by $\cos \delta$, we can calculate the average angular offset $\bar{\delta}_{\text {avg,i }}$ using the average velocities derived with the LSQ and LTS method and equ. A.1 equ. A.2 and equ. A.3.

$$
\operatorname{bias}=\frac{1}{N} \sum_{i=1}^{N}\left(v_{C S S, i}-v_{S C, i}\right)=\overline{v_{\mathrm{avg}, \mathrm{CSS}}}-\overline{v_{\mathrm{avg}, \mathrm{SC}}}
$$

$$
\cos \bar{\delta}_{\mathrm{avg}, \mathrm{i}}=\frac{\bar{v}_{\mathrm{avg}, \mathrm{CSS}, \mathrm{i}}}{\bar{v}_{\mathrm{avg}, \mathrm{CSS}, \mathrm{i}}-\mathrm{bias}}
$$

For the LSQ method with an average speed of $8.61 \mathrm{~m} / \mathrm{s}$ and a bias of $0.21 \mathrm{~m} / \mathrm{s}$ for $\pm 5 \mathrm{~min}$ medians, an $\bar{\delta}_{\mathrm{avg}, \mathrm{LSQ}}=12.4^{\circ}$ is found $\left(\cos \bar{\delta}_{\mathrm{avg}, \mathrm{LSQ}}=\right.$ $0.977)$. For the LTS method ( \pm 5 min medians) with an average speed of $8.48 \mathrm{~m} / \mathrm{s}$ and a bias of $-0.42 \mathrm{~m} / \mathrm{s}$, an $\bar{\delta}_{\text {avg,LTS }}=17.8^{\circ}$ is found $\left(\cos \bar{\delta}_{\text {avg,LTS }}=0.952\right)$. However, as we can see in the previous section on one example day, the bias is not completely caused by $\delta$. Therefore, this effect is arguably not of outmost importance or hidden behind other deviations.

The correction made in the previous section and the bias correction made here could only be accomplished using a reference measurement system. Several approaches are possible to make such a correction without reference measurements and will be studied in the following.

\section{A.2.1 Calculate corrections factors based on cloud speeds}

A correction approach for $\cos \delta$ based on cloud speeds is discussed Wang et al. 2016, section 4.3), but could not be tested due to the lack of a reference system. Using the shadow camera measurements, this suggested correction is investigated in this section. The suggested approach can be made operational by using the maximum velocity measured during a given period of time for all corresponding measurements. The maximum velocity is thus considered to be $v_{\text {real }}$. Additionally, this velocity is considered to be perpendicular to the cloud edge. Both assumptions are questionable. 
Table A.1: Cloud speed deviations found for the LSQ and LTS approach with speed-derived corrections applied in comparison to the shadow camera measurements on 59 days.

\begin{tabular}{l|llll} 
& LSQ $_{ \pm 2 \text { min,corr,max }}$ & LSQ $_{ \pm 5 \text { min,corr,max }}$ & LTS $_{ \pm 2 \text { min,corr,max }}$ & LTS $_{ \pm 5 \text { min,corr,max }}$ \\
\hline RMSD & $3.1 \mathrm{~m} / \mathrm{s}(41.7 \%)$ & $3.7 \mathrm{~m} / \mathrm{s}(50.8 \%)$ & $3.9 \mathrm{~m} / \mathrm{s}(53.6 \%)$ & $4.7 \mathrm{~m} / \mathrm{s}(64.3 \%)$ \\
MAD & $1.8 \mathrm{~m} / \mathrm{s}(24.0 \%)$ & $2.1 \mathrm{~m} / \mathrm{s}(29.1 \%)$ & $2.4 \mathrm{~m} / \mathrm{s}(32.5 \%)$ & $3.0 \mathrm{~m} / \mathrm{s}(40.3 \%)$ \\
bias & $1.0 \mathrm{~m} / \mathrm{s}(+14.0 \%)$ & $1.6 \mathrm{~m} / \mathrm{s}(+22.9 \%)$ & $1.4 \mathrm{~m} / \mathrm{s}(+19.2 \%)$ & $2.4 \mathrm{~m} / \mathrm{s}(+32.0 \%)$
\end{tabular}

Table A.1 shows the deviations found if the maximum speed measured in a ${ }_{634}$ period of time is compared to the medians of the shadow camera for the same ${ }_{635}$ period. In comparison to Tab. 8 in which the deviations without this correction ${ }_{636}$ are presented, the deviations shown here are significantly larger. Especially the ${ }_{637}$ bias, which is now positive, is increased by this correction. The larger deviations ${ }_{638}$ are caused by the scatter present in the CSS measurements (visualized in the ${ }_{639}$ plots of section 4.1). Moreover, cloud speeds might change significantly within ${ }_{640} \pm 5 \mathrm{~min}$. Thus, this correction approach is not feasible.

\section{${ }_{641}$ A.2.2 Calculate corrections factors based on cloud directions}

642 Another approach to derive correction factors for cloud speeds not perpen643 dicular to the corresponding cloud edges is based on the directions. For a period ${ }_{644}$ of time, a median cloud motion direction is calculated. This way, $\cos \delta$ can be 645 estimated for every measurement and the velocities can be corrected. Thus ${ }_{646}$ derived, $\delta$ is Gaussian distribution with a standard deviation of e.g. $52.8^{\circ}$ for ${ }_{647} \mathrm{LSQ}_{ \pm 2 \text { min,corr }}$.

In Tab. A.2 the deviations in comparison to the shadow camera measure649 ments are shown. Offsets greater than one standard deviation are not corrected. ${ }_{650}$ Including these corrections leads to higher deviations. The velocities are not fur${ }_{651}$ ther temporally averaged within the considered time periods.

${ }_{652}$ In comparison to Tab. 8 Tab. A.2 shows higher deviations. Increasing the 653 period of time to calculate the median cloud motion vectors from $\pm 2 \mathrm{~min}$ to ${ }_{654} \pm 5$ min increases the RMSD and MAD. Notably, the bias is reduced. In sum${ }_{655}$ mary, we conclude that this correction approach is not feasible. The reason for 
Table A.2: Cloud speed deviations found for the LSQ and LTS approach with direction-derived corrections applied in comparison to the shadow camera measurements on 59 days. $\delta$ above one standard deviation are not corrected. The velocities are not further temporally averaged within the considered time periods.

\begin{tabular}{|c|c|c|c|c|}
\hline & $\mathrm{LSQ}_{ \pm 2 \text { min,corr }}$ & $\mathrm{LSQ}_{ \pm 5 \text { min,corr }}$ & $\mathrm{LTS}_{ \pm 2 \text { min,corr }}$ & $\operatorname{LTS}_{ \pm 5 \text { min,corr }}$ \\
\hline RMSD & $2.8 \mathrm{~m} / \mathrm{s}(37.7 \%)$ & $2.8 \mathrm{~m} / \mathrm{s}(37.6 \%)$ & $3.5 \mathrm{~m} / \mathrm{s}(47.6 \%)$ & $3.6 \mathrm{~m} / \mathrm{s}(49.3 \%)$ \\
\hline MAD & $1.6 \mathrm{~m} / \mathrm{s}(22.4 \%)$ & $1.7 \mathrm{~m} / \mathrm{s}(22.8 \%)$ & $2.1 \mathrm{~m} / \mathrm{s}(28.9 \%)$ & $2.2 \mathrm{~m} / \mathrm{s}(30.3 \%)$ \\
\hline bias & $+0.1 \mathrm{~m} / \mathrm{s}(+1.2 \%)$ & $+0.2 \mathrm{~m} / \mathrm{s}(2.5 \%)$ & $-0.1 \mathrm{~m} / \mathrm{s}(-1.2 \%)$ & $-0.02 \mathrm{~m} / \mathrm{s}(-0.3 \%)$ \\
\hline
\end{tabular}

${ }_{656}$ this is, similar as discussed in the previous section, the scatter of the CSS mea-

${ }_{657}$ surements. Furthermore, it is a mere assumption that the median cloud motion

${ }_{658}$ vector itself is perpendicular to the cloud edge.

\section{A.2.3 Assuming circular clouds}

The angular offset $\delta$ can be corrected if the shape and the size of each cloud

${ }_{661}$ is known. In this section, this approach is investigated by calculating backwards

${ }_{662}$ using $\bar{\delta}_{\text {avg,i }}$ derived earlier at the beginning of section A.2 Figure A.3 visualizes

${ }_{663}$ the situation. A circular cloud with unkown radius $\mathrm{R}$ is approaching the CSS

${ }_{664}$ from one particular direction. The distance D is $29.7 \mathrm{~cm}$ (see Fig. 1b) and we use

${ }_{665} \delta=\bar{\delta}_{\mathrm{avg}, \mathrm{LSQ}}=12.4^{\circ}$ for this example calculation. Thus, $\mathrm{x}$ can be derived to be

$6666.5 \mathrm{~cm}$. $\beta$ is defined by $\sin \beta=\frac{D}{R}$ and $\cos \beta=\frac{R-x}{R}$. Using $(\sin \beta)^{2}+(\cos \beta)^{2}=1$,

${ }_{667}$ the radius can be determined to be $\mathrm{R}=70.8 \mathrm{~cm}$. Hypothetically, this radius may

668 correspond to intra-cloud structures and is far too small for usual cloud sizes. As

${ }_{669}$ both the shape (here: circular) and the size of the clouds/intra-cloud structures

670 must be assumed to achieve this correction, this approach is not feasible. The

671 calculations shown in this section are included for further understanding of the 672 general problem. 


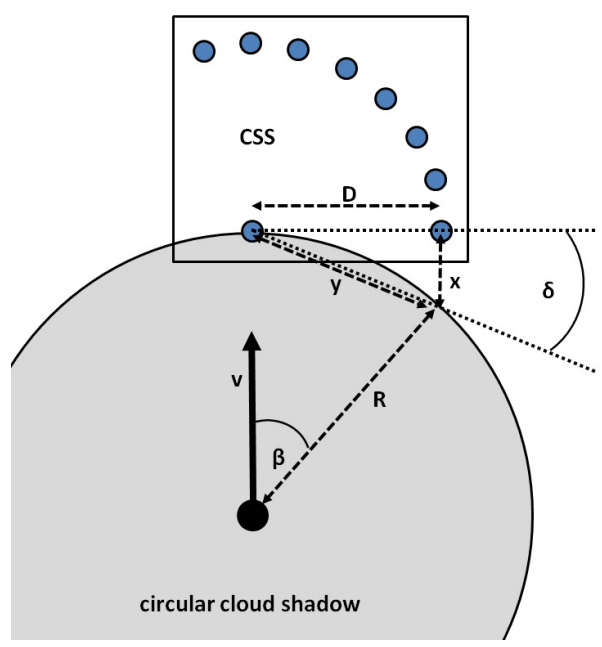

Figure A.3: Visualization of the circular cloud assumption to correct $\delta$.

${ }_{684}$ Bloomfield, P., Steiger, W., 2012. Least absolute deviations: Theory, applica-

Here, a fundamental challenge within the linear cloud edge - curve fitting method was studied and several correction approaches investigated. All considered correction approaches increase deviations in comparison to shadow camera reference measurements. It was found that in general, the deviations caused by non-perpendicular cloud motion vectors are, at least for the weather conditions considered here, of minor importance. We therefore suggest not to apply the presented correction approaches. However, we like to stress that these offsets must be kept in mind.

\section{References}

\section{References}

\section{A.3 Concluding remarks: Linear cloud edge - curve fitting method}

685 tions and algorithms. volume 6. Springer Science \& Business Media.

${ }_{686}$ Bosch, J.L., Kleissl, J., 2013. Cloud motion vectors from a network of ground 687 sensors in a solar power plant. Solar Energy 95, 13-20. URL: http://dx.doi. 


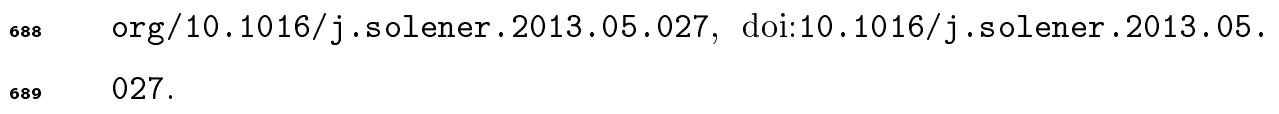

Bosch, J.L., Zheng, Y., Kleissl, J., 2013. Deriving cloud velocity from an array of solar radiation measurements. Solar Energy 87, 196-

692 203. URL: http://dx.doi.org/10.1016/j.solener.2012.10.020 doi:10.

$693 \quad 1016 / \mathrm{j}$.solener.2012.10.020.

${ }_{694}$ Chen, X., Du, Y., Wen, H., 2017. Forecasting based power ramp-rate control for PV systems without energy storage, in: 2017 IEEE 3rd International Future Energy Electronics Conference and ECCE Asia (IFEEC 2017 -

697 ECCE Asia), pp. 733-738. URL: http://ieeexplore.ieee.org/abstract/ document/7992130/, doi:10.1109/IFEEC.2017.7992130

${ }_{699}$ Fung, V., Bosch, J.L., Roberts, S.W., Kleissl, J., 2013. Cloud shadow speed sensor. Atmospheric Measurement Techniques 6. URL:http://dx.doi.org/ 10.5194/amt-7-1693-2014, doi:10.5194/amt-7-1693-2014

Giloni, A., Padberg, M., 2002. Least Trimmed Squares Regression, Least Median Squares Regression, and Mathematical Programming. Mathematical and Computer Modelling 35, 1043-1060. URL: http://dx.doi.org/10.1016/ S0895-7177(02)00069-9, doi 10.1016/S0895-7177(02)00069-9

Hanrieder, N., Sengupta, M., Xie, Y., Wilbert, S., Pitz-Paal, R., 2016. Modeling beam attenuation in solar tower plants using common DNI measurements. Solar Energy 129, 244-255. URL: http://www.sciencedirect.com/science/ article/pii/S0038092X1600075X.

Huang, H., Yoo, S., Yu, D., Huang, D., Qin, H., 2012. Correlation and local feature based cloud motion estimation, in: Proceedings of the Twelfth International Workshop on Multimedia Data Mining, ACM, New York, NY, USA. pp. 1-9. URL: http://doi.acm.org/10.1145/2343862.2343863 doi: $10.1145 / 2343862.2343863$

Huber, P.J., 2009. Robust Statistics. Springer. 
Killius, N., Prahl, C., Hanrieder, N., Wilbert, S., Schroedter-Homscheidt, M., 2015. On the use of NWP for Cloud Base Height Estimation in Cloud CameraBased Solar Irradiance Nowcasting. URL: http://www . wemcouncil.org/wp/ wp-content/uploads/2015/07/1510_MarionSchroedterHomscheidt .pdf oral presenation on EUPVSEC.

Klein, S.A., Jakob, C., 1999. Validation and Sensitivities of Frontal Clouds Simulated by the ECMWF Model. Monthly Weather Review 127, 2514-2531. URL: http://journals.ametsoc.org/doi/abs/10.1175/ 1520-0493(1999) 127\%3C2514\%3AVASOFC\%3E2.0.C0\%3B2.

Kuhn, P., Nouri, B., Wilbert, S., Prahl, C., Kozonek, N., Schmidt, T., Yasser, Z., Ramirez, L., Zarzalejo, L., Meyer, A., Vuilleumier, L., Heinemann, D., Blanc, P., Pitz-Paal, R., 2017a. Validation of an all-sky imager-based nowcasting system for industrial PV plants. Progress in Photovoltaics: Research and Applications , n/a-n/aURL: http://dx.doi.org/10.1002/pip.2968 doi:10.1002/pip.2968. pIP-17-122.R1.

Kuhn, P., Wilbert, S., Prahl, C., Garsche, D., Schüler, D., Haase, T., Ramirez, L., Zarzalejo, L., Meyer, A., Blanc, P., Pitz-Paal, R., 2018a. Applications of a shadow camera system for energy meteorology. Advances in Science and Research 15, 11-14. URL: https://www.adv-sci-res.net/15/11/2018/ doi:10.5194/asr-15-11-2018.

Kuhn, P., Wilbert, S., Prahl, C., Schüler, D., Haase, T., Hirsch, T., Wittmann, M., Ramirez, L., Zerzalejo, L., Meyer, A., Vuilleumier, L., Blanc, P., Pitz-Paal, R., 2017b. Shadow camera system for the generation of solar irradiance maps. Solar Energy 157, 157 - 170. URL: http://www.sciencedirect.com/science/article/pii/ S0038092X17304814, doi:https://doi.org/10.1016/j.solener.2017.05. 074

Kuhn, P., Wilbert, S., Schüler, D., Prahl, C., Haase, T., Ramirez, L., Zarzalejo, L., Meyer, A., Vuilleumier, L., Blanc, P., Dubrana, J., Kazantzidis, 
A., Schroedter-Homscheidt, M., Hirsch, T., Pitz-Paal, R., 2017c. Validation of spatially resolved all sky imager derived DNI nowcasts. AIP Conference Proceedings 1850, 140014. URL: http://aip.scitation.org/doi/abs/10. $1063 / 1.4984522$, doi $10.1063 / 1.4984522$

Kuhn, P., Wirtz, M., Killius, N., Wilbert, S., Bosch, J., Hanrieder, N., Nouri, B., Kleissl, J., Ramirez, L., Schroedter-Homscheidt, M., Heinemann, D., Kazantzidis, A., Blanc, P., Pitz-Paal, R., 2018b. Benchmarking three low-cost, low-maintenance cloud height measurement systems and ECMWF cloud heights against a ceilometer. Solar Energy , -URL: https://www.sciencedirect.com/science/article/pii/ S0038092X1830183X, doi:https://doi.org/10.1016/j.solener.2018.02. 050 .

Lappalainen, K., Valkealahti, S., 2016a. Analysis of shading periods caused by moving clouds. Solar Energy 135, 188-196. URL: http://dx.doi.org/10. 1016/j.solener.2016.05.050, doi:10.1016/j.solener.2016.05.050

Lappalainen, K., Valkealahti, S., 2016b. Apparent velocity of shadow edges caused by moving clouds. Solar Energy 138, 47-52. URL: http://dx.doi. org/10.1016/j.solener.2016.09.008, doi:10.1016/j.solener.2016.09. 008 .

Lave, M., Kleissl, J., Ellis, A., Mejia, F., 2013. Simulated PV power plant variability: Impact of utility-imposed ramp limitations in Puerto Rico, in: 2013 IEEE 39th Photovoltaic Specialists Conference (PVSC), pp. 18171821. URL: http://ieeexplore.ieee.org/document/6744495/, doi:10. 1109/PVSC.2013.6744495

Mäki, A., Valkealahti, S., 2012. Power Losses in Long String and ParallelConnected Short Strings of Series-Connected Silicon-Based Photovoltaic Modules Due to Partial Shading Conditions. IEEE Transactions on Energy Conversion 27, 173-183. URL: http://dx.doi.org/10.1109/TEC.2011. 2175928, doi:10.1109/TEC.2011.2175928. 
774 Marcos, J., 1, O.S., Marroyo, L., Garcia, M., Lorenzo, E., 2014. Storage requirements for PV power ramp-rate control. Solar Energy 99, 28 - 35. URL: http://www.sciencedirect.com/science/article/ pii/S0038092X13004672, doi/http://doi.org/10.1016/j.solener.2013. 10.037 .

Molteni, F., Buizza, R., Palmer, T.N., Petroliagis, T., 1996. The ECMWF Ensemble Prediction System: Methodology and validation. Quarterly Journal of the Royal Meteorological Society 122, 73-119. URL: http://dx.doi.org/ 10.1002/qj.49712252905, doi:10.1002/qj . 49712252905.

Mount, D.M., Netanyahu, N.S., Piatko, C.D., Silverman, R., Wu, A.Y., 2014. On the Least Trimmed Squares Estimator. Algorithmica 69, 148-183. URL: http://dx.doi.org/10.1007/s00453-012-9721-8, doi:10. 1007/s00453-012-9721-8

Rousseeuw, P.J., 1984. Least median of squares regression. Journal of the American statistical association 79, 871-880. URL: http://dx.doi.org/10. $2307 / 2288718$ doi $10.2307 / 2288718$.

Rousseeuw, P.J., Croux, C., 1993. Alternatives to the median absolute deviation. Journal of the American Statistical association 88, 1273-1283. URL: http://dx.doi.org/10.1080/01621459.1993.10476408 doi:10.1080/01621459.1993.10476408

Scaramuzza, D., Martinelli, A., Siegwart, R., 2006. A toolbox for easily calibrating omnidirectional cameras, in: 2006 IEEE/RSJ International Conference on Intelligent Robots and Systems, IEEE. pp. 5695-5701. URL: http:// ieeexplore.iee.org/abstract/document/4059340/, doi:10.1109/IR0S. 2006.282372

Schenk, H., Hirsch, T., Wittmann, M., Wilbert, S., Keller, L., Prahl, C., 2015. Design and Operation of an Irradiance Measurement Network. Energy Procedia 69, 2019-2030. URL: http://dx.doi.org/10.1016/j . egypro. 2015. 03.212 doi $10.1016 / \mathrm{j}$.egypro.2015.03.212. 
${ }_{803}$ Tomassini, M., Kelly, G., Saunders, R., 1999. Use and Impact of Satellite

${ }_{804}$ Atmospheric Motion Winds on ECMWF Analyses and Forecasts. Monthly

805 \Weather Review 127, 971-986. URL: http://journals.ametsoc.org/doi/

so6 full/10.1175/1520-0493(1999) 127\%3C0971\%3AUAIOSA\%3E2.0.CO\%3B2.

${ }_{807}$ Wang, G., Kurtz, B., Kleissl, J., 2016. Cloud base height from sky imager and 808 \ cloud speed sensor. Solar Energy 131, 208-221. URL: http://dx.doi.org/

so9 $10.1016 / j \cdot$ solener.2016.02.027, doi $10.1016 / j . s o l e n e r .2016 .02 .027$. 